\title{
Targeting the two-pore channel 2 in cancer progression and metastasis
}

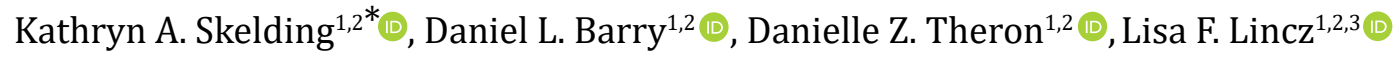 \\ ${ }^{1}$ Cancer Cell Biology Research Group, School of Biomedical Sciences and Pharmacy, College of Health, Medicine and Wellbeing, \\ The University of Newcastle, Callaghan, New South Wales 2308, Australia \\ ${ }^{2}$ Hunter Medical Research Institute, New Lambton Heights, New South Wales 2305, Australia \\ ${ }^{3}$ Hunter Hematology Research Group, Calvary Mater Newcastle Hospital, Waratah, New South Wales 2298, Australia
}

*Correspondence: Kathryn A. Skelding, Cancer Cell Biology Research Group, School of Biomedical Sciences and Pharmacy, College of Health, Medicine and Wellbeing, The University of Newcastle, University Drive, Callaghan, New South Wales 2308, Australia. Kathryn.Skelding@newcastle.edu.au

Academic Editor: Zui Pan, The University of Texas at Arlington, USA

Received: December 17, 2021 Accepted: February 2, 2022 Published: February 28, 2022

Cite this article: Skelding KA, Barry DL, Theron DZ, Lincz LF. Targeting the two-pore channel 2 in cancer progression and metastasis. Explor Target Antitumor Ther. 2022;3:62-89. https://doi.org/10.37349/etat.2022.00072

\begin{abstract}
The importance of $\mathrm{Ca}^{2+}$ signaling, and particularly $\mathrm{Ca}^{2+}$ channels, in key events of cancer cell function such as proliferation, metastasis, autophagy and angiogenesis, has recently begun to be appreciated. Of particular note are two-pore channels (TPCs), a group of recently identified $\mathrm{Ca}^{2+}$-channels, located within the endolysosomal system. TPC2 has recently emerged as an intracellular ion channel of significant pathophysiological relevance, specifically in cancer, and interest in its role as an anti-cancer drug target has begun to be explored. Herein, an overview of the cancer-related functions of TPC2 and a discussion of its potential as a target for therapeutic intervention, including a summary of clinical trials examining the TPC2 inhibitors, naringenin, tetrandrine, and verapamil for the treatment of various cancers is provided.
\end{abstract}

\section{Keywords}

Two-pore channel 2, TPCN2, tetrandrine, naringenin, cancer, anti-cancer drugs, verapamil

\section{Introduction}

$\mathrm{Ca}^{2+}$ is a major second messenger in cells and alterations in intracellular $\mathrm{Ca}^{2+}$ signaling regulate a variety of biological processes, including exocytosis/endocytosis, cell proliferation, invasion, migration, and apoptosis. Dysregulation of $\mathrm{Ca}^{2+}$ signaling is increasingly being demonstrated to contribute to the development of cancer [1], and has been implicated in each of the hallmarks of cancer originally identified by Hanahan and Weinberg [2].

For the most part, alterations in intracellular $\mathrm{Ca}^{2+}$ occur due to the opening of $\mathrm{Ca}^{2+}$-channels. Many different types of $\mathrm{Ca}^{2+}$-channels are found in cells; some are expressed on the plasma membrane [e.g., voltage-gated $\mathrm{Ca}^{2+}$ channels, transient receptor potential (TRP) channels, and store-operated channels], whereas others are expressed on the membrane of intracellular organelles, such as ryanodine receptors (RyRs) and inositol 1,4,5-trisphosphate receptors ( $\mathrm{IP}_{3} \mathrm{R}$ ) located on the sarcoplasmic and endoplasmic

(C) The Author(s) 2022. This is an Open Access article licensed under a Creative Commons Attribution 4.0 International License (https://creativecommons.org/licenses/by/4.0/), which permits unrestricted use, sharing, adaptation, distribution and reproduction in any medium or format, for any purpose, even commercially, as long as you give appropriate credit to the original author(s) and the source, provide a link to the Creative Commons license, and indicate if changes were made. 
reticulum. Two-pore channels (TPCs) are unique in that they are located on the acidic organelles of the endolysosomal system [3].

The endolysosomal system is a complex pathway of dynamic organelles responsible for the delivery of cargo from the cell surface to internal lysosomes via trafficking through early endosomes and late endosomes. Final transfer to lysosomes is achieved by the fusion of late endosomes with lysosomes to form transient hybrid organelles known as endolysosomes, from which lysosomes are eventually reformed. These acidic, protease-laden organelles were referred to as "suicide bags" by the scientist who discovered them [4], making them attractive targets for cancer therapies that could permeabilize their membranes to allow toxic hydrolases to escape into the cytosol. But it is now known that the endosomal network is used for a multitude of cellular functions, including recycling and cell signaling, cell death and survival, and ultimately maintaining cell homoeostasis [5]. Thus, the seemingly simple concept of lysosome membrane destabilization as a therapeutic option has taken on more complexity, but also offers more potential.

The recently identified TPC2 (also known as TPCN2) $\mathrm{Ca}^{2+}$-channel provides the seemingly perfect anticancer target. Although the full range of TPC2 functions are only beginning to be understood, it has already emerged as an intracellular ion channel of significant pathophysiological relevance, including in a variety of cancer-related functions. This review will summarize developments in our understanding of TPC2 in pathophysiological conditions, with a focus on cancer, and highlight the pre-clinical evidence for targeting TPCs as a novel anti-cancer therapeutic strategy.

\section{TPC structure and activation}

TPCs comprise a family of voltage- and ligand-gated $\mathrm{Na}^{+} / \mathrm{Ca}^{2+}$ ion channels exclusively located in endolysosomes. TPC1 and 3 are voltage-gated channels, whereas TPC2 opens in response to binding phosphatidylinositol-3,5-diphosphate $\left[\mathrm{PI}(3,5) \mathrm{P}_{2}\right]$ and nicotinic acid adenine dinucleotide phosphate (NAADP) [6-8]. However, the role of TPC2 in NAADP-mediated $\mathrm{Ca}^{2+}$ release has been controversial $[9,10]$. In an effort to resolve this controversy, Jha et al. [11] identified regulators of TPC2, and demonstrated that $\mathrm{Mg}^{2+}$ can inhibit these NAADP-mediated $\mathrm{Ca}^{2+}$ currents, but that when $\mathrm{Mg}^{2+}$ is absent, NAADP can activate TPC2, which may account for the conflicting findings.
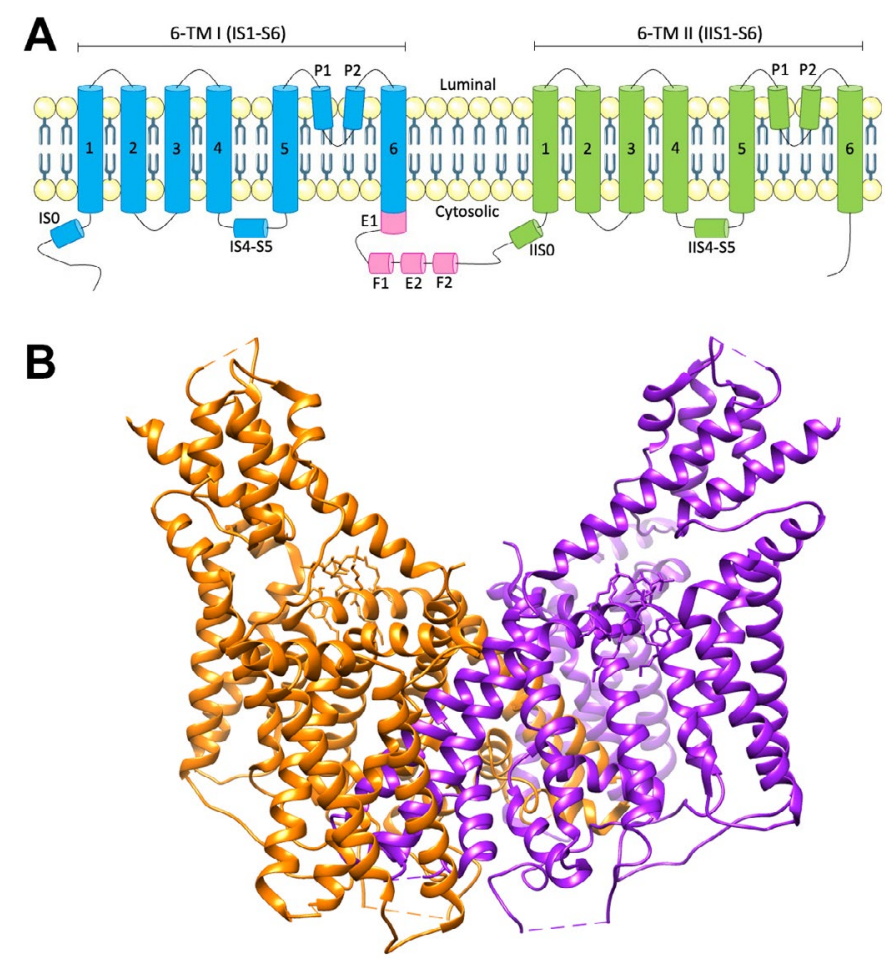

Figure 1. Schematic of TPC2 structure. A) Topology and domain arrangement of a human TPC2 subunit; B) crystal structure of human TPC2. PDB: 6NQ2. Generated using Chimera [17]. The two different protein subunits are shown in orange and purple. E F: EF-hand motifs; P: pore domain; TM: transmembrane 
While three distantly related genes (TPC1-3) have been identified, humans and rodents only express two isoforms, TPC1 and TPC2 [12], encoded by genes in humans located on chromosomes 12 and 11, respectively. TPC2 expression is localized to the late endosome and lysosomal membranes, whereas TPC1 shows a broader distribution throughout the endolysosomal system, particularly in earlier, less acidic endosomal compartments $[7,13]$. While the name "TPC" suggests an ion channel with two separate pores, TPCs actually consist of two subunits forming a dimer. Each TPC subunit is comprised of two homologous shaker-like six transmembrane domain repeats (labeled IS1-S6 and IIS1-S6) (Figure 1), with the two-pore loop domains located in IS5-S6 and IIS5-S6 (Figure 1). PI(3,5) $\mathrm{P}_{2}$ binds to the first 6 transmembrane domains to activate the channel independently of voltage changes, inducing a structural change at IS6 [14]. However, the binding region of NAADP remains unknown. TPC1 and TPC2 subunits form homo- and hetero-dimers, with four pore domains forming the central channel in a two-fold symmetric arrangement $[15,16]$.

\section{TPC interactome}

While NAADP can endogenously regulate $\mathrm{Ca}^{2+}$ release via TPC2, how it does so remains unknown, and it has been suggested that a separate unidentified NAADP-binding protein acts to accessorize TPC2 activation. As a result, the identification of NAADP-TPC2 interactomes is currently an area of great interest (Figure 2), and proteomic characterisation has unsurprisingly revealed that TPC2 complexes with proteins involved in $\mathrm{Ca}^{2+}$ homeostasis, trafficking and membrane organisation, including scaffold Rab GTPases, where Rab binding was essential for NAADP-evoked TPC2 $\mathrm{Ca}^{2+}$ release [18]. TPC2 can also associate with the transient receptor potential mucolipin 1 (TRPML1) ion channel, but despite this interaction, TRPML1 and TPC2 function as independent ion channels [19]. Although the physiological relevance of this interaction remains unknown, it is most likely related to the control of lysosomal pH. Additionally, TPC2 can associate with the leucinerich repeat kinase 2 (LRRK2), mutations in which are linked to late-onset Parkinson's disease [20], and the mammalian target of rapamycin (mTOR), a serine/threonine protein kinase that when hyperactivated leads to increased growth and proliferation [10]. These findings suggest that TPC2 may be involved in the pathological processes of both Parkinson's disease and cancer. Silencing of endogenous expression of TPC2, but not TPC1, reduces store-operated $\mathrm{Ca}^{2+}$ entry (SOCE), which is modulated through the endoplasmic reticulum $\mathrm{Ca}^{2+}$ sensor stromal interaction molecule-1 (STIM1) and plasma membrane Orai1 $\mathrm{Ca}^{2+}$ channels, both of which were found to associate with TPC2 [21]. A yeast 2 hybrid assay identified hematopoietic cell-specific protein 1 (HS1)-associated protein X-1 (Hax-1) as a novel binding partner for both TPC1 and TPC2 [22]. Hax-1 has recently been implicated in apoptotic signaling [23], and the TPC/Hax-1 interaction may potentially play a role in modulating apoptosis. A recent study has identified a largely uncharacterized Sm-like protein Lsm12 complexed with NAADP, TPC1, and TPC2 [24], thus potentially identifying the missing accessory protein that has been postulated to exist for NAADP-mediated activation of TPC2. A better understanding of the TPC interactome may potentially lead to the identification of effective pharmacological modulators of TPC-mediated cellular functions, which will have broad implications for a variety of disease processes.

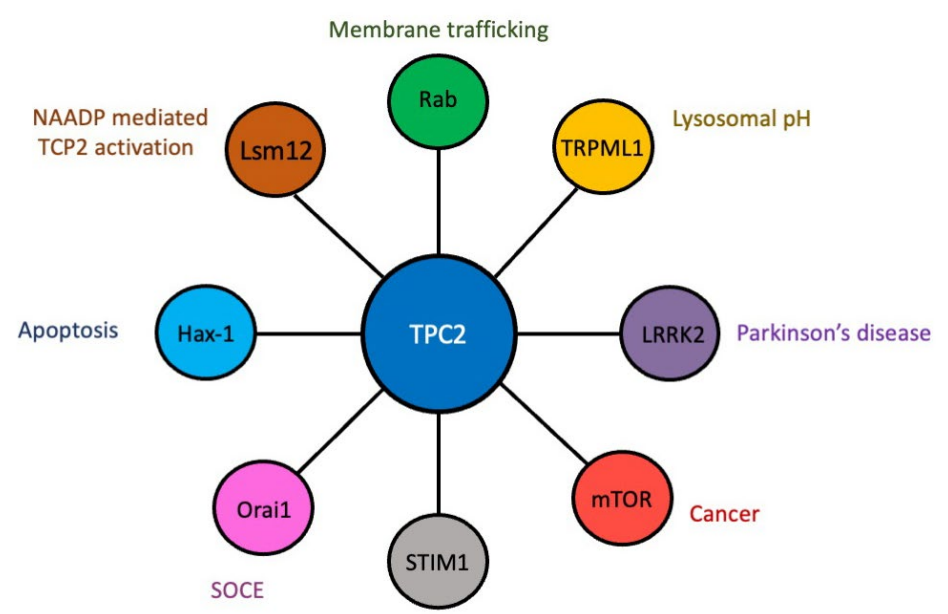

Figure 2. The TPC2 interactome. Diagrammatic representation of proteins that complex with TPC2 and the cellular/ physiological processes in which they are known to play a role 


\section{TPC2 functions}

TPC2 plays important roles in various diseases and physiological conditions, including cell proliferation, differentiation, development, autophagy, membrane trafficking, endolysosomal degradation pathway, phagocytosis, angiogenesis, low-density lipoprotein (LDL)-cholesterol trafficking, $\mathrm{T}$ cell activation, hormone secretion from the pancreas, and cardiac function [25-34]. Consequently, NAADP/TPC2/Ca ${ }^{2+}$ signaling has been shown to play a critical role in a variety of associated pathophysiological processes, including the life cycle of Ebola virus, human immunodeficiency virus (HIV), Middle East respiratory syndrome coronavirus (MERS-CoV), and severe acute respiratory syndrome coronavirus 2 (SARS-CoV-2), Parkinson's disease, Alzheimer's disease, non-alcoholic fatty liver disease, and cardiac dysfunction [28, 31, 35-42], thus highlighting the importance of the endolysosomal system in a variety of pathophysiological processes. Importantly, pharmacological inhibitors of TPC2 are potentially beneficial for the treatment of Ebola virus, SARS-CoV-2, Parkinson's disease, and Alzheimer's disease [35, 40, 41, 43], highlighting that TPC2 may be a suitable drug target for a plethora of pathophysiological processes.

Of particular note, TPC2 is overexpressed or mutated in several cancer types. Additionally, TPC2 has recently been implicated in a variety of cancer-related processes (such as proliferation, adhesion, migration, invasion, angiogenesis, and autophagy) that underpin the key hallmarks of cancer [2] (Figure 3).

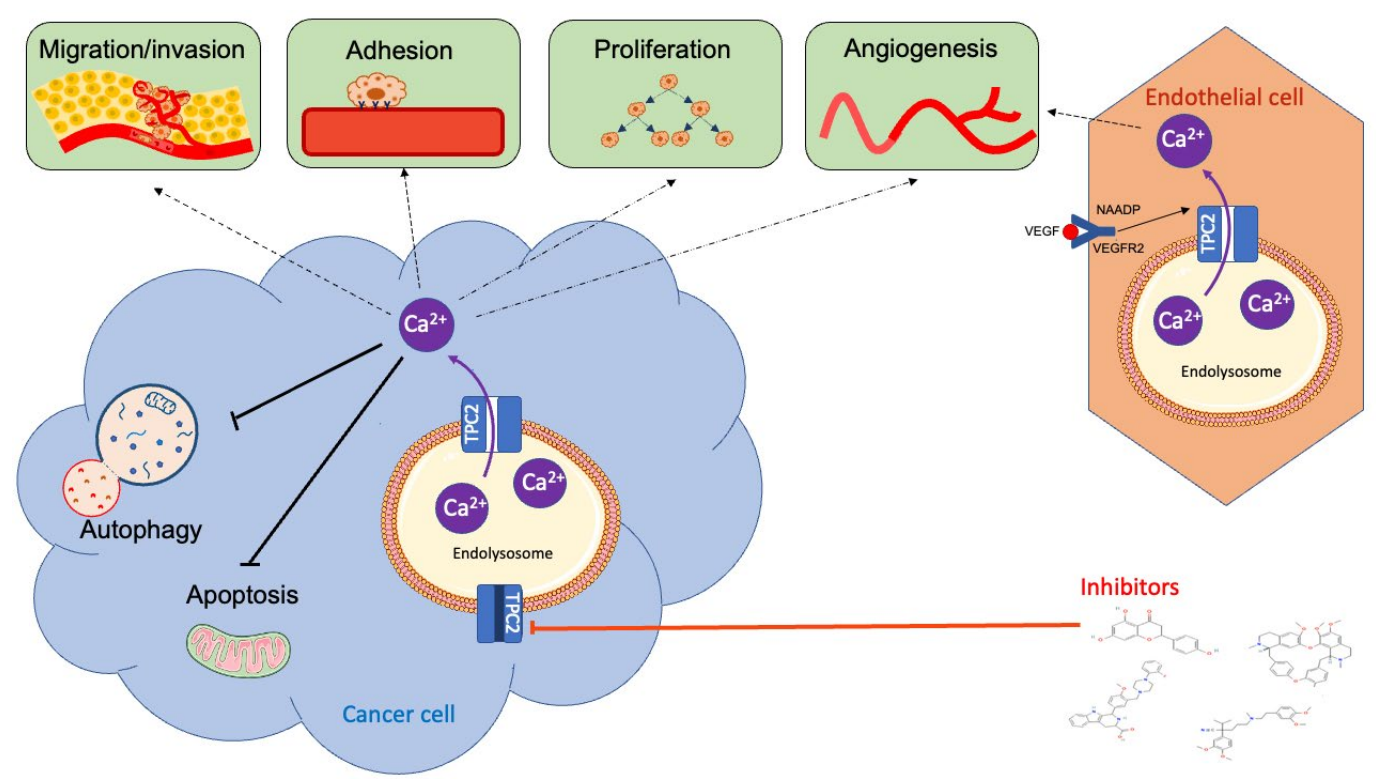

Figure 3. Overview of the role of TPC2 in cancer-related processes. The TPC2/NAADP/Ca ${ }^{2+}$ signaling pathway has been implicated in proliferation, apoptosis, adhesion, invasion, migration, autophagy, and angiogenesis in vitro and in vivo. Several TPC2 pharmacological inhibitors, including tetrandrine, verapamil, Ned-19 and naringenin, have been demonstrated to inhibit these cancer-related processes in vitro and in vivo. VEGF: vascular endothelial growth factor; VEGFR2: vascular endothelial growth factor receptor 2

\section{TPC2 function in cancer-related processes}

\section{Cell growth and differentiation}

A role for TPC2 in proliferation was first postulated based on the finding that TPC2 function could be regulated by the protein kinases, p38 mitogen activated protein kinase (MAPK), c-jun N-terminal kinase (JNK), and the mTOR complex 1 (mTORC1) [11]. Since then, TPC2 has been directly implicated in controlling cancer cell growth and differentiation in a variety of different cell types. Knockdown of TPC2 expression in pulmonary artery smooth muscle cells, MNT-1 human melanoma cells, RIL175 murine hepatocellular carcinoma cells, and 4T1 murine breast cancer cells significantly decreased proliferation in vitro [29, 44-46], demonstrating that TPC2 can control both normal and cancerous cell proliferation. Additionally, decreasing TPC2 expression in hepatocellular carcinoma cells reduced glycolysis and respiratory activity in vitro [45], potentially accounting for these proliferative effects, and completely abrogated hepatocellular carcinoma tumor growth in vivo [45]. Further, knockout of the TPCN2 gene in murine primary melanoma cells decreased 
initial proliferation rates by slowing $\mathrm{G}_{2}$ phase progression [47]. By contrast, TPC2 overexpression in 4T1 breast cancer cells did not influence proliferation [29], suggesting that this control of proliferation may not be related to the level of TPC2 expression, but rather to the presence of TPC2. Taken together, these results indicate that TPC2 controls cancer cell proliferation highlighting that it may be a useful target for controlling cancer cell growth.

Changes in TPC2 expression have been observed at various stages in differentiation in multiple cell types, suggesting that TPC2 may control differentiation. For example, TPC2 mRNA expression was highest in the first 3 days of differentiation of C2C12 myoblast cells but was reduced thereafter, and TPC2 knockdown inhibited differentiation of these myoblasts [48]. Similarly, TPC2 mRNA was increased in osteoclast precursor cells upon receptor activator of nuclear factor kappa B (NFKB) ligand (RANKL)induced osteoclast differentiation, and downregulation of TPC2 suppressed osteoclastogenesis [49]. By contrast, in mouse day 3 embryonic stem cells, TPC2 was markedly decreased during the early stages of neural differentiation but increased at later times during neuronal differentiation [50]. As a result, TPC2 knockdown accelerated the initial commitment of neural progenitors, but inhibited later neuronal differentiation. Additionally, overexpression of TPC2 induced cell death and prevented embryonic stem cells from differentiating into early neural lineages [50]. Interestingly, TPC2 knockdown had no effect on the differentiation of astrocytes or oligodendrocytes [50]. Taken together, these studies demonstrate that the role of TPC2 in promoting differentiation is cell type specific.

\section{Cancer cell migration, invasion, and metastasis}

Emerging evidence has also implicated TPC2 in all the major steps of tumor metastasis, namely adhesion, migration, and invasion. Knockdown of TPC2 expression in T24 bladder cancer and CHL1 and B16-F10 melanoma cells, and pharmacological inhibition in T24, HUH7 liver cancer and 4T1 breast cancer cells, significantly reduced adhesion [47, 51]. Mechanistically, the knockout of the TPC2 gene in CHL1 and B16-F10 melanoma cell lines decreased adhesion to collagen type I matrix and was associated with a decrease in $\alpha 2 \beta 1$ integrin expression on the plasma membrane [47]. While pharmacological inhibition resulting in reduced adhesion to collagen type I matrix may initially appear to be counterintuitive and to promote metastasis, several studies have shown that metastasis is instead promoted by increased adhesion of mda- 9 / syntenin protein to collage type I and activation of $\beta 1$ integrin signaling complexes [52]. Whether decreasing adhesion to collagen via pharmacological inhibition of TPC2 promotes or inhibits metastasis remains to be seen, highlighting that the future application of pharmacological inhibitors of TPC2 needs to be cautiously evaluated to ensure that they do not promote metastasis in patients.

In support of TPC2 having a metastasis promoting role, knockdown of TPC2 expression in MNT-2 melanoma cells, T24 bladder cancer cells, RIL175 hepatocellular carcinoma, 4T1 breast cancer, Hela cervical cancer and pulmonary artery smooth muscle cells, and pharmacological inhibition in T24, HUH7 and 4T1 breast cancer cells led to decreased migration in vitro [29, 44-46, 51]. Mechanistically, the downregulation of TPC2 resulted in accumulation of enlarged acidic vesicles, as general recycling was impaired, which halted $\beta 1$-integrin trafficking, and prevented leading-edge formation [51], providing further support that pharmacological inhibition of TPC2-mediated inhibition of $\beta 1$-integrin signaling will prevent, rather than promote, metastasis. By contrast, TPC2 overexpression in 4T1 breast cancer cells had no effect on migration in vitro [29]. Taken together, these studies suggest that TPC2 can control cancer cell migration in vitro, however, similarly as to what was observed for proliferation, the presence of TPC2, rather than an increased level of expression, may be the only requirement for the regulation of migration.

TPC2 has also been implicated in invasion in melanoma cells. Knockout of TPC2 in MNT-1 human melanoma cells significantly reduced invasion in vitro [44], providing further support for TPC2 possessing a metastasis promoting role. By contrast, knockout of the TPC2 gene in CHL1 and B16-F10 primary murine melanoma cells increased invasion, with an associated increase in matrix metalloproteinase 9 (MMP-9) expression [47]. These conflicting studies highlight that additional examination of the precise role of TPC2 in cancer, particularly melanoma, is required to identify whether TPC2 promotes or prevents invasion and metastasis. 
Taken together, these in vitro studies demonstrate the importance of TPC2 in adhesion, migration, and invasion, and suggest that TPC2 may promote metastasis in vivo. Indeed, knockdown of TPC2 expression and pharmacological inhibition in 4T1 orthotopic breast cancer and RIL175 hepatocellular carcinoma mouse xenograft models reduced the formation of lung metastases in vivo [45, 51], providing additional evidence that TPC2 is an important enhancer of metastasis in vivo, particularly for breast and hepatocellular cancer, and suggest that TPC2 may be a potential therapeutic target for preventing cancer metastasis, at least in breast and hepatocellular cancers. Because the expression levels of TPC2 seem to be less important than its presence for most cancer-associated functions, further investigation into TPC2 binding partners in specific cancer cell types will ultimately be required to unravel the pathways responsible for the varying effects observed.

\section{Angiogenesis}

Tumor cells are highly dependent on the formation of new blood vessels to sustain their insatiable growth requirements. This pathological process of neoangiogenesis involves stimulation of normally quiescent endothelial cells through their VEGF receptors to induce proliferation, migration, and capillary branch formation. Production of VEGF by tumor cells is the key mediator of this process, but VEGF also affects immune cells in the tumor micro-environment as well as providing an autocrine pro-malignancy signal that promotes epithelial-mesenchymal transition of the cancer cells [53]. VEGF inhibitors initially appeared promising as anti-cancer therapies but were ultimately overcome by tumor cells that developed compensatory ways to survive [54]. The discovery that signaling through the VEGFR2 receptor subtype involves NAADP and TPC2-dependent lysosomal $\mathrm{Ca}^{2+}$ release [33] offers alternative strategies for targeting this pathway. In these studies, TPC2, but not TPC1, knockdown in endothelial cells inhibited VEGF-induced $\mathrm{Ca}^{2+}$ release and angiogenesis in vitro [33]. Further, pharmacological inhibition of TPC2 decreased angiogenesis in glioma and breast cancer models in vivo [55-57]. Additionally, Tpcn2 ${ }^{\%}$ mice or pharmacological inhibition of TPC2 inhibited vascularisation of VEGF-containing matrigel plugs in vivo $[33,58]$. TPC2 has also been implicated in controlling VEGF-dependent neovascularization in a mouse model of age-related macular degeneration [59], demonstrating that these angiogenic pathways are likely to be a normal response to VEGF-VEGFR2 signaling in endothelial cells. In contrast, autocrine VEGF signaling in cancer cells is expected to be dysregulated through alterations in TPC2 function. VEGFR2 is expressed by a multitude of cancers, ranging from solid tumors (such as brain, gynaecological, gastrointestinal, lung) to those of the haematopoietic system (multiple myeloma, myeloid leukaemias) [3]. Thus, pharmacological targeting of TPC2 will have many effects on many cell types, with the potential to alter both the tumor and its microenvironment.

\section{Autophagy}

Autophagy is a lysosomal dependent mechanism used to naturally rid the cell of dysfunctional or redundant elements and is a critical regulator of cellular homeostasis. In extreme cases of starvation, a cell will use autophagy as a source of energy to maintain survival, and defects in this pathway have been associated with numerous human diseases, including cancer [60]. However, autophagy plays a dual role in cancer, where it can play a tumor suppressive role in normal tissue but promote tumorigenesis in cancer tissue, by increasing proliferation, angiogenesis, metastasis, and decreasing apoptosis [61]. The lysosomal location of TPC2 implies involvement in autophagic pathways, but this is enigmatic and there is debate as to whether TPC2 promotes or hampers autophagy.

Several studies have indicated that TPC2 is a positive regulator of autophagic flux. Overexpression of TPC2 in astrocytes increased the expression of the autophagic markers LC3-II and beclin-1, as well as acidic vesicular organelle formation [62]. Conversely, expression of a dominant-negative TPC2 construct led to decreased LC3-II levels in these astrocytes, and knockdown of TPC2 expression decreased autophagy in astrocytes in an mTOR-independent manner [63]. Furthermore, in cardiomyocytes, starvation induced a significant increase in TPC1 and TPC2 expression that paralleled increased autophagy [64]. Additionally, silencing of TPC2 alone or in combination with TPC1 in nutrient-rich conditions induced autophagy, 
whereas under starvation, silencing of either TPC1 or TPC2 induced an autophagic block [64]. This study suggests that TPC2 is mainly required for basal autophagy, while both TPC1 and TPC2 are required for starvation-induced autophagy. Additionally, one of the identified TPC2 binding partners, LRRK2, is a regulator of autophagy, involving activation of the calcium/calmodulin-stimulated protein kinase kinase $\beta$ (CaMKK $\beta$ )/adenosine monophosphate (AMP)-activated protein kinase (AMPK) pathway [20]. These LRRK2mediated effects on autophagy could be completely abrogated by the expression of a dominant-negative, but not wild-type, TPC2 construct [20], identifying a potential functional role for the LRRK2/TPC2 interaction.

By contrast, there is also evidence that TPC2 can act as a negative regulator of autophagic flux. Overexpression of TPC2, but not an inactive mutant form of TPC2, in Hela cervical cancer and 4T1 breast cancer cells decreased autophagosomal-lysosomal fusion, resulting in the accumulation of autophagosomes [29, 65]. Conversely, TPC2 knockdown in mouse embryonic stem cells promoted autophagosomal-lysosomal fusion [65], and autophagy-related gene 5 (ATG5) knockdown, but not mTOR activity inhibition, abolished the TPC2-induced accumulation of autophagosomes [65]. Further, skeletal muscles from Tpcn2\% mice exhibited enhanced autophagy flux characterized by increased accumulation of autophagosomes upon starvation and treatment with the microtubule inhibitor, colchicine [66]. Taken together, the studies demonstrating a positive regulation of autophagy effect for TPC2 have largely involved examination in normal cells, whereas the studies showing a negative regulation of autophagy mostly investigated cancer or embryonic stem cells, suggesting that the cell type will influence the TPC2-mediated effect on autophagy.

Further controversy surrounds the mechanisms by which TPC2 may mediate autophagy. While the TPC-mediated effects in astrocytes occurred in an mTOR-independent manner [63], pharmacological inhibition of TPC2 in HGC-27 gastric cancer, T24 and 5637 bladder cancer cells and MDA-MB-231 breast cancer cells induced autophagy by inhibiting mTOR signaling pathway [67-69], suggesting that TPC2mediated positive regulation of autophagy in normal cells may occur in an mTOR-independent manner, but that TPC-mediated negative regulation of autophagy may occur in an mTOR-dependent manner.

\section{TPC2 alterations and expression in cancer}

As TPC2 has been implicated in several cancer related processes, it raises the question as to whether abnormalities in TPC2 gene coding or protein expression may be observed in cancer. Inherited polymorphisms in TPC2 have been identified as predisposition factors for a range of cancer types [70-72], most notably melanoma due to the role of TPC2 in controlling pigmentation through regulation of melanosomes [73]. However, acquired abnormalities within the TPC2 coding region appear restricted to rare instances of gene fusions in breast cancer and uterine leiomyomas [74, 75]. The chromosomal region harbouring TPC2 (11q13.2) is commonly amplified in cancer [76], and overexpression of the TPC2 gene has been identified as the potential driver of this amplification by providing growth advantage in oral squamous cell carcinoma [77, 78]. Hepatocellular carcinoma patient samples show highly positive staining for TPC2, and the majority of breast cancer and non-tumorigenic breast samples express TPC2 [51, 79]. Additionally, increased expression of TPC2 has been observed in cell lines derived from blood, liver and bladder cancer relative to MDA-MB-231 breast cancer cells [51].

TPC2 expression is also a potential prognostic biomarker for several types of solid tumors. TPC2 was part of a gene signature for biomarkers of lymph node metastasis in oral squamous cell carcinoma, recurrence in prostate cancer, and decreased overall survival in bladder cancer [77, 80, 81], and has been associated with increased risk of melanoma [82]. Taken together, these studies indicate that TPC2 is highly expressed in hepatocellular carcinoma, oral squamous cell carcinoma and blood cancers, however, whether this expression is observed in additional cancer types, remains to be seen. Additionally, increased TPC2 expression may be a biomarker for poor prognosis for several types of cancer, including oral, prostate and bladder cancers. These negative effects on patient prognosis are most likely due to the central role of TPC2 in regulating cancer cell proliferation and metastasis and highlight that inhibiting TPC2 activity is likely to be an anti-cancer therapeutic strategy for a range of cancer types. 


\section{Pharmacological inhibition of TPC as an anti-cancer strategy}

Due to the importance of TPC2 in controlling cancer-related functions (Figure 3), its suitability as an anti-cancer target is beginning to be appreciated. Several pharmacological inhibitors of TPC2 have been identified (Table 1) and their pre-clinical efficacy in a range of cancer types has recently begun to be explored (Table 2).

Table 1. Summary of pharmacological inhibitors of TPC2 that have been pre-clinically evaluated in cancer

\begin{tabular}{|c|c|c|}
\hline Name & Class & Structure \\
\hline Naringenin & Flavonoid & \\
\hline Tetrandrine & $\mathrm{Ca}^{2+}$ channel blocker & \\
\hline Ned-19 & NAADP-antagonist & \\
\hline Verapamil & $\mathrm{Ca}^{2+}$ channel blocker & \\
\hline
\end{tabular}

Structures obtained from PubChem [83].

Note. Structure image of Naringenin is reprinted from https://pubchem.ncbi.nlm.nih.gov/compound/932\#section=2D-Structure; structure image of Tetrandrine is reprinted from https://pubchem.ncbi.nlm.nih.gov/compound/73078\#section=2D-Structure; structure image of Ned-19 is reprint from https://pubchem.ncbi.nlm.nih.gov/compound/3978027\#section=2D-Structure; structure image of Verapamil is adapted from https://pubchem.ncbi.nIm.nih.gov/compound/2520\#section=2D-Structure

\section{Naringenin}

Naringenin, a natural flavonoid found in citrus and tomatoes, is one of the main flavonoids present in the human diet [84]. Naringenin has a wide range of potential applications, including anti-viral, anti-ageing, antiinflammatory, lipid lowering, anti-microbial, blood thinning and cardioprotective properties [84]. Anecdotally, higher serum concentrations of flavonoids, including naringenin, are significantly associated with lower breast, lung, and gastric cancer risk [85-89], suggesting that naringenin may possess anti-cancer properties. Mechanistically, naringenin can inhibit TPC2 and dampen the NAADP-stimulated $\mathrm{Ca}^{2+}$ response [58], and as such has been explored as a TPC2 inhibitor. However, naringenin also has other non-TPC2 related actions, and is predicted to act as an estrogen receptor $\alpha(E R \alpha)$ agonist [90], so its effects cannot be attributed solely to TPC2 inhibition.

Naringenin exhibits anti-proliferative and pro-apoptotic effects in glioma, melanoma, breast, colorectal, hepatic, prostate, lung, and pancreatic cancer in vitro [91-114], and in glioma, melanoma, breast, colorectal, hepatic, and lung cancer xenografts in vivo [91, 96-98, 115-121] (Table 2). By contrast, HCT116, HT29, and T84 colorectal cancer cells were only slightly sensitive to naringenin treatment in vitro but combined with 5-fluorouracil synergistically enhanced cell death [122]. Additionally, combining naringenin with paclitaxel 
synergistically increased cytotoxicity in prostate cancer cells in vitro [123], and when combined with histone deacetylase inhibitors, synergistically suppressed neuroblastoma tumor progression in vivo [124]. Taken together, these studies suggest that naringenin can act as a chemosensitiser. Indeed, naringenin has been demonstrated to alleviate multidrug resistance against gemcitabine and anthracyclines in a range of cancer types $[106,125]$. It is likely that naringenin will be most beneficial clinically in a combinatorial setting to alleviate multi-drug resistance in advanced cancers.

Table 2. Summary of pre-clinical evaluation of pharmacological inhibitors of TPC2, including proposed down-stream mechanisms

\begin{tabular}{|c|c|c|c|c|c|c|c|}
\hline \multirow[t]{2}{*}{ Cancer } & \multirow[t]{2}{*}{ Cell line(s) } & \multirow[b]{2}{*}{ Growth } & \multicolumn{2}{|c|}{ Effects } & \multirow[b]{2}{*}{$\begin{array}{l}\text { Growth in } \\
\text { vivo }\end{array}$} & \multirow[t]{2}{*}{ Mechanism } & \multirow[t]{2}{*}{ Ref } \\
\hline & & & $\begin{array}{l}\text { Cell } \\
\text { death }\end{array}$ & $\begin{array}{l}\text { Migration } \\
\& \text { invasion } \\
\end{array}$ & & & \\
\hline \multicolumn{8}{|l|}{ Naringenin } \\
\hline \multirow[t]{4}{*}{ Glioma } & $\mathrm{C} 6$ & - & - & - & $\downarrow$ & $\begin{array}{l}\text { ROS, cyclin D1, NFKB, } \\
\text { CDK4 }\end{array}$ & [118] \\
\hline & $\mathrm{C} 6$ & - & - & - & $\downarrow$ & PI3K, PKB & [119] \\
\hline & U-118MG & - & $\uparrow$ & - & - & - & [108] \\
\hline & GBM8401 & None & $\uparrow$ & $\downarrow$ & - & MMP & [109] \\
\hline \multirow[t]{4}{*}{ Melanoma } & B16F10 & $\downarrow$ & $\uparrow$ & - & $\downarrow$ (lung mets) & Transglutaminase & [110] \\
\hline & B16F10 & $\downarrow$ & $\uparrow$ & - & - & - & [111] \\
\hline & $\begin{array}{l}\text { B16F10, SK- } \\
\text { MEL-28 }\end{array}$ & $\downarrow$ & $\uparrow$ & $\downarrow$ & - & $\begin{array}{l}\text { ERK } 1 / 2 \text {, JNK, MAPK, } \\
\text { PARP, caspase }\end{array}$ & [112] \\
\hline & B16F10 & - & - & - & $\downarrow$ & TGF $\beta-S m a d-M M P 2$ & [120] \\
\hline \multirow[t]{10}{*}{ Breast cancer } & MDA-MB-435 & $\downarrow$ & - & - & $\begin{array}{l}\downarrow \text { (delayed } \\
\text { tumor growth; } \\
\text { DMBA rat) }\end{array}$ & - & [91] \\
\hline & $4 \mathrm{~T} 1$ & None & - & - & $\downarrow$ (lung mets) & IFN-y, IL-2 & [126] \\
\hline & $\begin{array}{l}\text { MCF-7, T47D, } \\
\text { MDA-MB-231 }\end{array}$ & $\downarrow$ & $\uparrow$ & - & - & Caspase, p38 & [92] \\
\hline & $\begin{array}{l}\text { MDA-MB-231, } \\
\text { MDA-MB-468 }\end{array}$ & - & $\uparrow$ & - & - & - & [93] \\
\hline & HTB26, HTB132 & $\downarrow$ & $\uparrow$ & - & - & $\begin{array}{l}\text { Cyclins, caspases, PI3K/ } \\
\text { Akt pathway, NFKB }\end{array}$ & [94] \\
\hline & $\begin{array}{l}\text { SKBR3, MDA- } \\
\text { MB-231 }\end{array}$ & $\downarrow$ & $\uparrow$ & - & - & HER2 & [95] \\
\hline & $4 \mathrm{~T} 1$ & - & - & - & $\downarrow$ (lung mets) & TGF $\beta$ & [127] \\
\hline & E0771 & $\downarrow$ & $\uparrow$ & - & $\begin{array}{l}\downarrow \text { (delayed } \\
\text { tumor growth) }\end{array}$ & AMPK, cyclin D1 & [96] \\
\hline & MDA-MB-231 & $\downarrow$ & $\uparrow$ & $\downarrow$ & $\downarrow$ & $\begin{array}{l}\text { Mitochondria, NFKB, } \\
\text { biotransformation } \\
\text { enzymes }\end{array}$ & [97] \\
\hline & MCF-7, T47D & - & $\uparrow$ & - & $\downarrow$ & Aromatase & [98] \\
\hline \multirow[t]{7}{*}{$\begin{array}{l}\text { Colorectal } \\
\text { cancer }\end{array}$} & $\begin{array}{l}\text { Azoxymethane rat } \\
\text { model }\end{array}$ & - & - & - & $\downarrow$ & - & [115] \\
\hline & HCT116 & $\downarrow$ & - & - & - & $\begin{array}{l}\text { Cell cycle regulatory } \\
\text { protein expression }\end{array}$ & [99] \\
\hline & SW1116, SW837 & $\downarrow$ & $\uparrow$ & - & - & $\begin{array}{l}\text { Cyclins, caspases, PI3K/ } \\
\text { Akt pathway, NFKB }\end{array}$ & [94] \\
\hline & HCT116, SW480 & $\downarrow$ & - & - & - & Cyclin D1, p38 & [100] \\
\hline & HT29 & $\downarrow$ & $\uparrow$ & - & - & $\begin{array}{l}\text { Cell cycle and death } \\
\text { pathways }\end{array}$ & [101] \\
\hline & Caco-2 & $\downarrow$ & - & - & - & ROS & [102] \\
\hline & $\begin{array}{l}\text { HCT116, HT29, } \\
\text { T84 }\end{array}$ & None & None & - & - & MAPK & [122] \\
\hline
\end{tabular}


Table 2. Summary of pre-clinical evaluation of pharmacological inhibitors of TPC2, including proposed down-stream mechanisms (continued)

\begin{tabular}{|c|c|c|c|c|c|c|c|}
\hline \multirow[t]{2}{*}{ Cancer } & \multirow[t]{2}{*}{ Cell line(s) } & \multirow[b]{2}{*}{ Growth } & \multicolumn{2}{|c|}{ Effects } & \multirow[b]{2}{*}{$\begin{array}{l}\text { Growth in } \\
\text { vivo }\end{array}$} & \multirow[t]{2}{*}{ Mechanism } & \multirow[t]{2}{*}{ Ref } \\
\hline & & & $\begin{array}{l}\text { Cell } \\
\text { death }\end{array}$ & $\begin{array}{l}\text { Migration } \\
\text { \& invasion } \\
\end{array}$ & & & \\
\hline \multirow{3}{*}{$\begin{array}{l}\text { Hepatic } \\
\text { carcinoma }\end{array}$} & HepG2 & $\downarrow$ & $\uparrow$ & - & - & P53, caspase & [103] \\
\hline & $\begin{array}{l}\text { NDEA-induced rat } \\
\text { model }\end{array}$ & - & - & - & $\downarrow$ & $\begin{array}{l}\text { PCNA, BCl-2, NFKB, } \\
\text { VEGF, MMP }\end{array}$ & [116] \\
\hline & $\begin{array}{l}\text { NDEA-induced rat } \\
\text { model }\end{array}$ & - & - & - & $\downarrow$ & Antioxidant & [117] \\
\hline \multirow{3}{*}{ Prostate cancer } & PC3, LnCaP & $\downarrow$ & $\uparrow$ & $\downarrow$ & - & ROS, mitochondria & [104] \\
\hline & $\mathrm{PC} 3$ & - & - & $\downarrow$ & - & EMT, uPA activity & [128] \\
\hline & Mat-LyLu & $\downarrow$ & - & $\downarrow$ & - & SCN9A & [105] \\
\hline \multirow{2}{*}{$\begin{array}{l}\text { Pancreatic } \\
\text { cancer }\end{array}$} & ASPC-1, PANC-1 & - & $\uparrow$ & $\downarrow$ & - & EMT & [106] \\
\hline & SNU-213 & - & $\uparrow$ & - & - & ROS & [107] \\
\hline \multirow[t]{4}{*}{ Lung cancer } & NRG mice model & - & - & - & $\downarrow$ & CYP1A1, NFKB, PCNA & [121] \\
\hline & A549 & $\downarrow$ & - & $\downarrow$ & - & MMP-2/9, Akt & [113] \\
\hline & A549 & $\downarrow$ & $\uparrow$ & $\downarrow$ & - & Caspase, MMP & [114] \\
\hline & LLC & - & - & - & $\downarrow$ & TGF $\beta-S m a d-M M P 2$ & [120] \\
\hline \multicolumn{8}{|l|}{ Tetrandrine } \\
\hline \multirow[t]{4}{*}{ AML } & U937 & $\downarrow$ & $\uparrow$ & - & - & Caspase, JNK, PKC- $\delta$ & [129] \\
\hline & NB4 & $\downarrow$ & - & - & $\downarrow$ & ROS, Notch-1 & [130] \\
\hline & $\begin{array}{l}\text { K562, } 6133 \\
\text { MPLW515L }\end{array}$ & $\downarrow$ & $\uparrow$ & - & - & $\begin{array}{l}\text { P21, p27, ROS, Notch-1 } \\
\text { signaling }\end{array}$ & [131] \\
\hline & $\begin{array}{l}\text { HL60, K562, } \\
\text { U937, THP-1 }\end{array}$ & $\downarrow$ & $\uparrow$ & - & $\downarrow$ & ROS, c-myc & [132] \\
\hline \multirow[t]{6}{*}{ Glioma } & RT-2 & $\downarrow$ & $\uparrow$ & - & $\downarrow$ & - & [55] \\
\hline & U-87 & $\downarrow$ & $\uparrow$ & $\downarrow$ & - & $\begin{array}{l}\text { ADAM17, PI3K/Akt } \\
\text { signaling pathway }\end{array}$ & [133] \\
\hline & $\begin{array}{l}\text { U-87, U251, SWO- } \\
38\end{array}$ & $\downarrow$ & $\uparrow$ & - & $\downarrow$ & STAT3 & [56] \\
\hline & U-87, U251 & $\begin{array}{l}\downarrow \\
\text { (Neurosphere } \\
\text { formation) }\end{array}$ & $\uparrow$ & - & - & $\beta$-catenin, PARP, Bcl-2 & [134] \\
\hline & U-87, U251 & $\downarrow$ & - & - & - & ERK & [135] \\
\hline & GBM 8401 & - & - & $\downarrow$ & - & $\begin{array}{l}\text { MMP-2/9, NFkB, Akt, } \\
\text { EGFR, E/N-cadherin }\end{array}$ & [136] \\
\hline \multirow[t]{2}{*}{ Neuroblastoma } & Neuro2a & $\downarrow$ & $\uparrow$ & - & - & ROS & [137] \\
\hline & SHSY5Y & - & $\uparrow$ & - & - & - & [138] \\
\hline \multirow[t]{2}{*}{ Osteosarcoma } & U-20S, MG-63 & - & $\uparrow$ & - & - & Apaf-1, Bid, Bax, Bcl-2 & [139] \\
\hline & $143 B$ & $\downarrow$ & $\uparrow$ & - & $\downarrow$ & PTEN, PCNA & [140] \\
\hline \multirow{2}{*}{$\begin{array}{l}\text { Nasopharyngeal } \\
\text { carcinoma }\end{array}$} & CNE & - & $\uparrow$ & - & - & $\mathrm{Bax} / \mathrm{Bcl}-2$ & [141] \\
\hline & NPC-TW076 & $\downarrow$ & $\uparrow$ & - & - & $\begin{array}{l}\text { Endoplasmic reticulum } \\
\text { stress }\end{array}$ & [142] \\
\hline \multirow[t]{2}{*}{ Lung cancer } & A549 & $\downarrow$ & $\uparrow$ & - & - & Akt, ERK & [143] \\
\hline & A549 & $\downarrow$ & $\uparrow$ & - & - & VEGF/HIF-1/ICAM-1 & [144] \\
\hline \multirow[t]{3}{*}{ Oral cancer } & SAS & - & $\uparrow$ & - & - & PARP, caspase & [145] \\
\hline & HSC-3 & - & $\uparrow$ & - & - & $\begin{array}{l}\text { PARP, caspase, beclin-1/ } \\
\text { LC3-1//l signaling }\end{array}$ & [146] \\
\hline & CAL27 & - & $\uparrow$ & - & - & $\begin{array}{l}\text { ROS, caspase, Beclin-1 } \\
\text { signaling }\end{array}$ & [147] \\
\hline \multirow[t]{2}{*}{ Gastric cancer } & BGC-823 & - & $\uparrow$ & - & - & $\begin{array}{l}\text { Caspase/mitochondria- } \\
\text { mediated }\end{array}$ & [148] \\
\hline & HGC-27 & - & $\uparrow$ & - & - & $\begin{array}{l}\text { PARP, caspase, Beclin-1/ } \\
\text { LC3-II/p62, Akt/mTOR }\end{array}$ & [67] \\
\hline
\end{tabular}


Table 2. Summary of pre-clinical evaluation of pharmacological inhibitors of TPC2, including proposed down-stream mechanisms (continued)

\begin{tabular}{|c|c|c|c|c|c|c|c|}
\hline \multirow[t]{2}{*}{ Cancer } & \multirow[t]{2}{*}{ Cell line(s) } & \multirow[b]{2}{*}{ Growth } & \multicolumn{2}{|c|}{ Effects } & \multirow[b]{2}{*}{$\begin{array}{l}\text { Growth in } \\
\text { vivo }\end{array}$} & \multirow[t]{2}{*}{ Mechanism } & \multirow[t]{2}{*}{ Ref } \\
\hline & & & $\begin{array}{l}\text { Cell } \\
\text { death }\end{array}$ & $\begin{array}{l}\text { Migration } \\
\text { \& invasion } \\
\end{array}$ & & & \\
\hline \multirow[t]{3}{*}{ Prostate cancer } & DU145, PC3 & $\downarrow$ & $\uparrow$ & - & - & ROS, JNK1/2 & [149] \\
\hline & DU145, PC3 & $\downarrow$ & $\uparrow$ & $\downarrow$ & - & PARP, PI3K/Akt & [150] \\
\hline & DU145, PC3 & - & - & $\downarrow$ & - & $\begin{array}{l}\text { Akt/mTOR/MMP-9 } \\
\text { signaling }\end{array}$ & [151] \\
\hline \multirow[t]{2}{*}{ Bladder cancer } & $5637, \mathrm{~T} 24$ & - & $\uparrow$ & - & - & $\begin{array}{l}\text { Caspase/mitochondria- } \\
\text { mediated }\end{array}$ & [152] \\
\hline & $5637, \mathrm{~T} 24$ & - & - & $\downarrow$ & - & $\begin{array}{l}\text { Inducing MET through } \\
\text { downregulation of Gli-1 }\end{array}$ & [153] \\
\hline \multirow[t]{5}{*}{ Breast cancer } & $\begin{array}{l}\text { SUM-149, SUM- } \\
\text { 159, sphere } \\
\text { (patient sample) }\end{array}$ & $\downarrow$ & - & - & - & - & [154] \\
\hline & $4 \mathrm{~T} 1$ & - & - & - & $\downarrow$ (lung mets) & $\begin{array}{l}\text { ERK, NFKB, VEGF, } \\
\text { HIF-1 } \alpha \text {, integrin } \beta 5 \text { and } \\
\text { ICAM-1 }\end{array}$ & {$[57]$} \\
\hline & MCF-7 & - & $\downarrow$ & - & - & PKCa, caspase & [155] \\
\hline & MDA-MB-231 & $\downarrow$ & $\uparrow$ & - & - & $\begin{array}{l}\text { Beclin-1/LC3-I/LC3-II and } \\
\text { PI3K/Akt/mTOR signaling } \\
\text { pathways }\end{array}$ & [68] \\
\hline & MDA-MB-231 & - & $\uparrow$ & - & $\downarrow$ & $\begin{array}{l}\text { Bcl-2, Bax, PARP, } \\
\text { caspase }\end{array}$ & {$[156]$} \\
\hline \multirow[t]{2}{*}{$\begin{array}{l}\text { Renal cell } \\
\text { carcinoma }\end{array}$} & $\begin{array}{l}\text { 786-O, 769-P, } \\
\text { ACHN }\end{array}$ & $\downarrow$ & $\uparrow$ & - & - & Caspase, p21 and p27 & [157] \\
\hline & 786-O, 769-P & - & - & $\downarrow$ & - & MMP-9, PI3K/Akt, NFKB & [158] \\
\hline \multirow{7}{*}{$\begin{array}{l}\text { Hepatic } \\
\text { carcinoma }\end{array}$} & HepG2 & $\downarrow$ & $\uparrow$ & - & - & Caspase & [159] \\
\hline & HepG2 & $\downarrow$ & $\uparrow$ & - & - & Caspase & [160] \\
\hline & $\begin{array}{l}\text { Hep G2, Hep 3B, } \\
\text { PLC/PRF/5 }\end{array}$ & $\downarrow$ & - & - & - & - & [161] \\
\hline & HepG2, Huh7 & - & $\uparrow$ & - & $\downarrow$ & ROS, Akt & [162] \\
\hline & Huh-7 & - & - & - & $\downarrow$ & ROS, ERK & [163] \\
\hline & Huh-7 & $\downarrow$ & $\uparrow$ & - & - & Caspase, PARP & [164] \\
\hline & $\begin{array}{l}\text { Huh7, SMMC- } \\
\text { 7721, HepG2, } \\
\text { PLC/PRF/5, } \\
\text { MHCC97H, SK- } \\
\text { Hep-1, SNU398 }\end{array}$ & $\downarrow$ & - & - & - & CaMKII & [165] \\
\hline \multirow{6}{*}{$\begin{array}{l}\text { Colorectal } \\
\text { cancer }\end{array}$} & CT-26 & - & $\uparrow$ & - & $\downarrow$ (lung mets) & - & [166] \\
\hline & HT-29 & $\downarrow$ & $\uparrow$ & - & - & PI3K/Akt/GSK3 $\beta$, PARP & [167] \\
\hline & CT-26 & - & $\uparrow$ & - & $\downarrow$ & P38 MAPK & [168] \\
\hline & SW480, HCT116 & $\downarrow$ & $\uparrow$ & $\downarrow$ & $\downarrow$ & Wnt/ß-catenin, & [169] \\
\hline & LoVo & $\downarrow$ & $\uparrow$ & - & $\downarrow$ & IGFBP-5, Wnt/B-catenin & {$[170]$} \\
\hline & НСТ116 & $\downarrow$ & $\uparrow$ & $\downarrow$ & - & MMP-2, EMT & [171] \\
\hline Cervical cancer & $\mathrm{SiHa}$ & $\downarrow$ & $\uparrow$ & $\downarrow$ & $\downarrow$ & $\begin{array}{l}\text { Caspase, MMP-2, } \\
\text { MMP-9 }\end{array}$ & {$[172]$} \\
\hline $\begin{array}{l}\text { Gallbladder } \\
\text { cancer }\end{array}$ & SGC-996 & $\downarrow$ & $\uparrow$ & - & - & $\begin{array}{l}\text { Caspase, PARP, } \\
\text { mitochondria }\end{array}$ & [173] \\
\hline $\begin{array}{l}\text { Pancreatic } \\
\text { cancer }\end{array}$ & $\mathrm{PaCa}$ & $\downarrow$ & - & - & $\downarrow$ & P21, p27, cyclin D & [174] \\
\hline Ovarian cancer & OVCAR-3, A2780 & $\downarrow$ & $\uparrow$ & - & $\downarrow$ & $\begin{array}{l}\text { Wht, E-cadherin, cyclin } \\
\text { D, c-myc }\end{array}$ & [175] \\
\hline \multicolumn{8}{|l|}{ Ned-19 } \\
\hline Bladder cancer & $\mathrm{T} 24$ & - & - & $\downarrow$ & - & Endocytic recycling & [51] \\
\hline
\end{tabular}


Table 2. Summary of pre-clinical evaluation of pharmacological inhibitors of TPC2, including proposed down-stream mechanisms (continued)

\begin{tabular}{|c|c|c|c|c|c|c|c|}
\hline \multirow[t]{2}{*}{ Cancer } & \multirow[t]{2}{*}{ Cell line(s) } & \multirow[b]{2}{*}{ Growth } & \multicolumn{2}{|c|}{ Effects } & & \multirow[t]{2}{*}{ Mechanism } & \multirow[t]{2}{*}{ Ref } \\
\hline & & & $\begin{array}{l}\text { Cell } \\
\text { death }\end{array}$ & $\begin{array}{l}\text { Migration } \\
\& \text { invasion } \\
\end{array}$ & $\begin{array}{l}\text { Growth in } \\
\text { vivo }\end{array}$ & & \\
\hline $\begin{array}{l}\text { Hepatic } \\
\text { carcinoma }\end{array}$ & Huh7 & - & - & $\downarrow$ & - & Endocytic recycling & [51] \\
\hline Breast cancer & $4 \mathrm{~T} 1$ & - & - & $\downarrow$ & $\downarrow$ & Endocytic recycling & [51] \\
\hline Melanoma & B16 & $\downarrow$ & $\uparrow$ & $\downarrow$ & $\downarrow$ & VEGF & [176] \\
\hline $\begin{array}{l}\text { Colorectal } \\
\text { cancer }\end{array}$ & Patient samples & $\downarrow$ & - & - & - & ERK, Akt & [177] \\
\hline \multicolumn{8}{|l|}{ Verapamil } \\
\hline Breast cancer & ZR-751A & $\uparrow$ & - & - & - & - & [178] \\
\hline $\begin{array}{l}\text { Colorectal } \\
\text { cancer }\end{array}$ & LoVo & $\uparrow$ & - & - & - & - & [178] \\
\hline \multirow[t]{2}{*}{ AML } & Patient samples & $\downarrow$ & - & - & - & - & [179] \\
\hline & Patient samples & $\downarrow$ & - & - & - & - & [180] \\
\hline CML & Patient samples & None & - & - & - & - & [180] \\
\hline
\end{tabular}

ADAM17: ADAM metallopeptidase domain 17; AML: acute myeloid leukaemia; Bcl-2: B-cell lymphoma-2; CaMKII: calcium/ calmodulin-stimulated protein kinase II; CDK4: cyclin-dependent kinase 4; CML: chronic myeloid leukaemia; DMBA: 7,12-dimethylbenz[a]anthracene; EGFR: epidermal growth factor receptor; EMT: epithelial to mesenchymal transition; ERK1: extracellular signal-regulated kinase 1; GSK3 $\beta$ : glycogen synthase kinase $3 \beta$; HER2: human epidermal growth factor receptor 2; HIF-1: hypoxia inducible factor 1; ICAM-1: intracellular adhesion molecule 1; IFN: interferon; IGFBP: insulin-like growth factor binding protein; IL-2: interleukin-2; MET: mesenchymal to epithelial transition; mets: metastases; NDEA: $N$-nitrosodiethylamine; PARP: polyadenosine-diphosphate-ribose polymerase; PCNA: proliferating cell nuclear antigen; PKB: protein kinase B; PI3K: phosphoinositide-3-kinase; PTEN: phosphatase and tensin homolog; Ref: reference; ROS: reactive oxygen species; SCN9A: sodium voltage-gated channel $\alpha$ subunit 9; STAT3: signal transducer and activator of transcription 3; TGF $\beta$ : transforming growth factor $\beta$; uPA: urokinase type plasminogen activator; $\downarrow$ : decreased effects; $\uparrow$ : increased or induced effects; -: not examined

Further, naringenin inhibits migration of glioma, melanoma, breast, prostate pancreatic, and lung cancer cells in vitro [97, 104-106, 109, 112-114, 128] (Table 2), suggesting that naringenin may act as an anti-metastatic agent, and that pharmacologically inhibiting TPC2 will inhibit, rather than promote metastasis. Indeed, naringenin can decrease lung metastases in 4T1 breast cancer and B16F10 melanoma xenografts in vivo [110,126, 127]. Anti-cancer agents that are effective against metastases are rare, and these pre-clinical findings highlight that naringenin, and inhibiting TPC2, may be a promising avenue for the treatment of metastatic cancers.

Several studies have investigated the mechanisms underlying naringenin-mediated anti-cancer effects and have identified a variety of mechanisms (Table 2), including immunomodulation, NFKB-signaling, ROS-mediated induction of apoptosis, PI3K/Akt pathway, and TGFß-mediated signaling pathways. Many of these pathways are known to be activated in cancer cells, thus providing additional support for naringenin being a suitable anti-cancer agent.

In an attempt to improve the effectiveness of naringenin, derivatives modified at position 7 have been developed. Initial examination has shown that HCT116 colon cancer cells were more sensitive to these derivatives than to naringenin [181], however, examination in additional clinically relevant laboratory models is required to ascertain whether cancer cells in general are more sensitive to these derivatives. Further, additional flavonoids from a Southeast Asian plant extract (Dalbergia parviflora) block TPC2 activity in melanoma cells and inhibit melanoma cell proliferation, migration, and invasion in vitro [44]. Further evaluation of these flavonoids is required; however, they may be an additional avenue of promising research.

Despite the abundance of pre-clinical evidence indicating that naringenin is a potent anti-cancer agent with low toxicity (Table 2), this has not been investigated clinically in cancer. Importantly, bioavailability of ingested natural compounds may be lower than required to exert an anti-cancer effect at a cellular level. However, clinical trials evaluating the safety and pharmacokinetics of naringenin in healthy adults have shown that naringenin ingestion had no related adverse events or alterations in blood safety 
markers [182]. Most promising, maximal blood concentrations of $48.45 \pm 7.88 \mu \mathrm{mol} / \mathrm{L}$ were achieved within $4 \mathrm{~h}$, with a half-life of 2-3 h. These concentrations are compatible with those required for inhibitory effects against cancer cells in vitro, with reported $\mathrm{IC}_{50}$ values ranging from 2.2-178 $\mu \mathrm{mol} / \mathrm{L}$ for most cell lines [183]. Future efforts should involve focusing on clinical trials of naringenin, both alone and as a chemosensitizing agent, in advanced cancers, particularly breast cancers.

\section{Tetrandrine}

Tetrandrine is isolated from the plant Stephania tetrandra and belongs to the class of bisbenzylisoquinoline alkaloids [184]. Tetrandrine has been used as a traditional medicine in China for decades to treat patients with autoimmune and inflammatory pulmonary disease, silicosis, cardiovascular diseases, and hypertension [185]. Tetrandrine acts as a $\mathrm{Ca}^{2+}$ channel blocker, and recently, tetrandrine was shown to block TPC2 currents elicited by both NAADP and $\mathrm{PI}(3,5) \mathrm{P}_{2}$ [35]. Additionally, tetrandrine inhibits the downstream regulator CaMKII [165], a kinase that has been implicated in cancer progression and metastasis $[1,186,187]$. This raises the question as to whether tetrandrine is blocking TPC2 itself or inhibiting downstream $\mathrm{Ca}^{2+}$-signaling pathways. Tetrandrine can also suppress another $\mathrm{Ca}^{2+}$-mediated process, specifically the nucleotide-binding domain, leucine-rich-repeat-containing family, pyrin domain-containing 3 (NLRP3) inflammasome activation via Sirt-1 $[188,189]$. Taken together, these studies highlight that tetrandrine is not a specific TPC2 inhibitor, but rather inhibits $\mathrm{Ca}^{2+}$-signaling related processes. Nevertheless, emerging evidence indicates that tetrandrine possesses anti-cancer efficacy against a range of cancer types (Table 2).

Tetrandrine displayed anti-proliferative and cytotoxic effects as a monotherapy against AML, glioma, neuroblastoma, osteosarcoma, nasopharyngeal, lung, oral, gastric, prostate, bladder, breast, renal cell, hepatocellular, colorectal, gallbladder, pancreatic, and ovarian cancer cells in vitro $[55,56,67,68,129-135$, 137-146, 148-150, 152, 154-157, 160-162, 164, 165, 167-175], and decreased tumor growth in glioma, osteosarcoma, AML, breast, hepatocellular, cervical, pancreatic, colorectal, and ovarian cancer xenograft models in vivo $[55,56,130,132,140,147,156,159,162,163,168,169,172,174,175]$ (Table 2). By contrast, despite nasopharyngeal cancer cells exhibiting sensitivity to tetrandrine in vitro, nasopharyngeal carcinoma xenografts were not sensitive to tetrandrine as a monotherapy in vivo, however tetrandrine was able to sensitise these tumors to irradiation [190], suggesting that it may act as a radiosensitising agent. Further, tetrandrine can also synergistically enhance the effects of numerous chemotherapeutics, including 5-flourouracil, imatinib, paclitaxel, vincristine, daunorubicin, cisplatin, sorafenib, arsenic trioxide, chloroquine, and the protein kinase inhibitor H89 both in vitro and in vivo [169, 175, 191-198], indicating that like naringenin, tetrandrine is also a chemosensitizing agent.

There is also substantial pre-clinical evidence that tetrandrine may be effective against metastatic disease. Tetrandrine treatment decreases invasion and migration of a range of cancer types in vitro $[51,133,134,136,150,151,153,158,169,171,172]$, and importantly, the formation of lung metastases in an orthotopic 4T1 breast cancer and an intravenous CT-26 colorectal cancer model in vivo [51, 57, 166] (Table 2), indicating that tetrandrine may be an effective agent for the treatment of metastatic disease.

Several studies have investigated the mechanisms underlying tetrandrine-mediated anti-cancer effects (Table 2). Although a variety of different mechanisms were identified, several were repeatedly observed, including ROS-mediated induction of apoptosis, PI3K/Akt/mTOR signaling, and caspase activation, suggesting that these may underpin the anti-proliferative and pro-apoptotic tetrandrine-mediated effects observed in a variety of cancer types.

Taken together, these studies highlight that tetrandrine is an interesting drug candidate for the treatment of cancer as a monotherapy and in combination with a variety of chemotherapeutics. While a large amount of pre-clinical evidence demonstrated that tetrandrine is effective in both in vitro and in vivo cancer models (Table 2), the majority of these studies were performed in two-dimensional cell culture models in only a single cancer cell line. Not all promising in vitro effects will translate into in vivo or clinical effects, therefore further examination in clinically relevant in vivo models is required before future 
clinical examination is performed. Additionally, it is likely that tetrandrine will be more beneficial clinically when combined with existing chemotherapeutics.

Nevertheless, a clinical trial examining the combination of intravenous tetrandrine with daunorubicin, etoposide and cytarabine for the treatment of refractory and relapsed AML showed that this combination was relatively well tolerated. Forty-two percent of patients achieved complete remission or restored chronic phase, 23\% achieved a partial response, and 34\% failed therapy [199], this provides the first evidence that tetrandrine may be a suitable therapy for the treatment of cancer. An additional clinical trial examined tetrandrine in combination with gemcitabine and cisplatin for patients with advanced non-small cell lung cancer. While the addition of tetrandrine to this regimen did not significantly improve the objective response rate, it did improve quality of life scores and mitigated adverse reactions to the chemotherapy [200], providing support for tetrandrine as a chemosensitiser.

Despite these promising results, several animal models have identified that tetrandrine can induce substantial liver and lung damage [201-203], which has raised concerns as to the use of tetrandrine in cancer patients. As such, analogues of tetrandrine, with lower toxicity profiles are under development [184]. Several of these analogues (RMS1-2, RMS4, RMS7-8) can inhibit proliferation and in some cases (RMS1, RMS4, RMS8) induce apoptosis of leukaemia cells that are resistant to a variety of chemotherapeutic drugs [184]. An additional analogue (H1) decreases proliferation and clonogenicity of colorectal, non-small cell lung cancer cells, and doxorubicin-resistant breast cancer cells in vitro [204-206]. Tetrandrine analogues that are potent TPC2 specific inhibitors have been identified by screening a library of synthesised benzyltetrahydroisoquinoline derivatives. One of these analogues, SG-094, suppresses tumor growth of RIL175 hepatocellular carcinoma xenografts in vivo [45]. While these initial results are promising, examination in additional more clinically relevant laboratory models is required.

\section{Ned-19}

Ned-19 is a selective membrane-permeable non-competitive NAADP antagonist [207], which likely inhibits TPCs in an indirect manner [208]. Ned-19 has primarily been used as an experimental tool to study the functions of TPCs, however, several studies have demonstrated pre-clinical efficacy for Ned-19 (Table 2). Ned-19 treatment decreased melanoma and colorectal cancer cell proliferation and induced apoptosis in B16 melanoma cells in vitro $[176,177]$. These studies further highlight the role of TPC2 in controlling important cancer-related functions and provide further evidence for the suitability of TPC2 as a potential anti-cancer target.

Further evidence indicates that Ned-19 may be a potential inhibitor of metastasis. Importantly, Ned-19 decreased migration of melanoma, hepatic, bladder, and breast cancer cells in vitro [51,177], and decreased the formation of lung metastases in 4T1 breast cancer and B16 melanoma xenograft models in vivo [51, 176]. While these results are promising, additional examination of Ned-19 is required before its clinical utility can be examined.

\section{Verapamil}

Verapamil is a $\mathrm{Ca}^{2+}$-channel blocker [209], used clinically to manage angina, arrhythmia and hypertension. Verapamil has also been shown to block NAADP-induced $\mathrm{Ca}^{2+}$ release [210], and hence antagonise TPCs. Based on the pre-clinical evidence for the other TPC inhibitors, it would be expected that verapamil also possesses anti-cancer properties. However, controversy exists concerning the potential association between $\mathrm{Ca}^{2+}$-channel blockers, particularly verapamil, and increased risk for the development of breast cancer [211]. Indeed, verapamil has a growth stimulatory effect on breast cancer cells in vitro [178], suggesting that it may indeed act as a tumor promoter for breast cancers. By contrast, verapamil inhibited proliferation ex vivo of tumor cells collected from AML, but not CML patients in blast phase $[179,180]$. Taken together, these studies highlight that verapamil exhibits dramatically different effects in different cell types, emphasising that a better understanding of the signaling pathways altered by verapamil in different cancer types is required. 
Despite these conflicting results on cancer cell proliferation, verapamil has been widely established to act as a chemosensitiser, by inhibiting the function of p-glycoprotein [212-214]. While in vitro evidence spanning several decades indicates that verapamil can sensitise a range of cancer cells to treatment with chemotherapeutics, including doxorubicin, daunorubicin, vincristine, paclitaxel, mitomycin and amlodipine [215-221], this efficacy has not always translated into improved survival benefit or tumor reduction in in vivo models. For example, verapamil + doxorubicin combination treatment did not increase survival or reduce metastatic burden in a 4T1-resistant breast cancer xenograft mouse model in vivo [215]. Additionally, orally administered verapamil significantly increased doxorubicin toxicity in mice [222]. Further, intravenous administration of verapamil in combination with doxorubicin and vincristine in patients with advanced and refractory breast cancer potentiated neurotoxicity and haemotoxicity [223]. By contrast, arterial infusion of verapamil alone and in combination with chemotherapeutics has been demonstrated to improve medical imaging parameters without increasing toxicity in primary liver cancer patients [224]. Taken together, these pre-clinical and clinical studies indicate that the route of administration of verapamil can influence the toxicity and chemosensitizing activity, and that arterial infusion of verapamil is the optimal clinical administration method. In support of this, targeted arterial perfusion of verapamil and chemotherapeutics (platinum therapy in combination with paclitaxel, docetaxel, 5-fluorouracil, and paclitaxel + gemcitabine + 5-fluorouracil) in advanced lung cancer patients [225] was clinically effective, where $85 \%$ of patients achieved a complete or partial remission, and all side-effects experienced with the combinatorial treatments resolved quickly [225]. In addition to being well tolerated, combining arterial verapamil with platinum and 5-fluorouracil and an anthracycline, or with docetaxel and anthracycline in advanced gastric cancer patients, significantly improved the efficacy over chemotherapy alone, and also improved progression-free and overall survival rates compared to chemotherapy alone [226]. These clinical studies identify that verapamil is particularly amenable to combination with platinum agents and also indicates that the route of administration of verapamil may impact its toxicity profile.

\section{Conclusion}

TPC2 is an attractive anti-cancer target as it is expressed in a range of cancer types and is vital in the control of cancer cell proliferation, survival, metastasis, and angiogenesis. Several drugs that inhibit TPC2, including the naturally occurring naringenin and tetrandrine, have been identified which demonstrate pre-clinical anti-cancer efficacy, including reducing metastatic burden in vivo. These agents have all been shown to act as chemosensitizing agents, suggesting that they will be most beneficial clinically when used in combination with existing chemotherapeutics. Indeed, several clinical studies have shown that tetrandrine and verapamil can potentiate the effects of chemotherapeutics in advanced cancer patients. However, both of these drugs can induce toxicity, most likely due to their $\mathrm{Ca}^{2+}$-channel blocking effects, so different routes of administration and analogues with improved safety profiles are being examined. Overall, a large body of evidence indicates that TPC2 is an attractive anti-cancer target that warrants further investigation.

\section{Abbreviations}

AML: acute myeloid leukaemia

Hax-1: hematopoietic cell-specific protein 1-associated protein X-1

JNK: c-jun N-terminal kinase

LRRK2: leucine-rich repeat kinase 2

MAPK: mitogen activated protein kinase

MMP-9: matrix metalloproteinase 9

mTOR: mammalian target of rapamycin

NAADP: nicotinic acid adenine dinucleotide phosphate

NFкB: nuclear factor kappa B

$\mathrm{PI}(3,5) \mathrm{P}_{2:}$ phosphatidylinositol-3,5-diphosphate 
PI3K: phosphoinositide-3-kinase

ROS: reactive oxygen species

TGF $\beta$ : transforming growth factor $\beta$

TPCs: two-pore channels

TRPML1: transient receptor potential mucolipin 1

VEGF: vascular endothelial growth factor

VEGFR2: vascular endothelial growth factor receptor 2

\section{Declarations}

Author contributions

All authors contributed to the drafting and editing of the manuscript. DLB and DZT created the figures. KAS and LFL contributed conception and design of the review. KAS, LFL, DLB and DZT contributed to manuscript revision, read, and approved the submitted version.

\section{Conflicts of interest}

The authors declare that they have no conflicts of interest.

Ethical approval

Not applicable.

\section{Consent to participate}

Not applicable.

\section{Consent to publication}

Not applicable.

\section{Availability of data and materials}

Not applicable.

\section{Funding}

Not applicable.

\section{Copyright}

(c) The Author(s) 2022.

\section{References}

1. Brzozowski JS, Skelding KA. The multi-functional calcium/calmodulin stimulated protein kinase (CaMK) family: emerging targets for anti-cancer therapeutic intervention. Pharmaceuticals (Basel). 2019;12:8.

2. Hanahan D, Weinberg RA. The hallmarks of cancer. Cell. 2000;100:57-70.

3. Zhu MX, Ma J, Parrington J, Calcraft PJ, Galione A, Evans AM. Calcium signaling via two-pore channels: local or global, that is the question. Am J Physiol Cell Physiol. 2010;298:C430-41.

4. de Duve C. Lysosomes, a new group of cytoplasmic particles. In: Hayashi T, editor. Subcellular particles. New York: Ronald Press Co;1959. pp. 129-59.

5. Repnik U, Cesen MH, Turk B. The endolysosomal system in cell death and survival. Cold Spring Harb Perspect Biol. 2013;5:a008755.

6. Kintzer AF, Stroud RM. On the structure and mechanism of two-pore channels. FEBS J. 2018;285:233-43. 
7. Calcraft PJ, Ruas M, Pan Z, Cheng X, Arredouani A, Hao X, et al. NAADP mobilizes calcium from acidic organelles through two-pore channels. Nature. 2009;459:596-600.

8. Zong X, Schieder M, Cuny H, Fenske S, Gruner C, Rotzer K, et al. The two-pore channel TPCN2 mediates NAADP-dependent $\mathrm{Ca}(2+)$-release from lysosomal stores. Pflugers Arch. 2009;458:891-9.

9. Wang X, Zhang X, Dong XP, Samie M, Li X, Cheng X, et al. TPC proteins are phosphoinositide- activated sodium-selective ion channels in endosomes and lysosomes. Cell. 2012;151:372-83.

10. Cang C, Zhou Y, Navarro B, Seo YJ, Aranda K, Shi L, et al. mTOR regulates lysosomal ATP-sensitive two-pore $\mathrm{Na(+)}$ channels to adapt to metabolic state. Cell. 2013;152:778-90.

11. Jha A, Ahuja M, Patel S, Brailoiu E, Muallem S. Convergent regulation of the lysosomal two-pore channel-2 by $\mathrm{Mg}^{2+}$, NAADP, PI(3,5) $\mathrm{P}_{2}$ and multiple protein kinases. EMBO J. 2014;33:501-11.

12. Brailoiu E, Rahman T, Churamani D, Prole DL, Brailoiu GC, Hooper R, et al. An NAADP-gated two-pore channel targeted to the plasma membrane uncouples triggering from amplifying $\mathrm{Ca}^{2+}$ signals. J Biol Chem. 2010;285:38511-6.

13. Brailoiu E, Churamani D, Cai X, Schrlau MG, Brailoiu GC, Gao X, et al. Essential requirement for two-pore channel 1 in NAADP-mediated calcium signaling. J Cell Biol. 2009;186:201-9.

14. She J, Zeng W, Guo J, Chen Q Bai XC, Jiang Y. Structural mechanisms of phospholipid activation of the human TPC2 channel. Elife. 2019;8:e45222.

15. Kintzer AF, Stroud RM. Structure, inhibition and regulation of two-pore channel TPC1 from Arabidopsis thaliana. Nature. 2016;531:258-62.

16. Rietdorf K, Funnell TM, Ruas M, Heinemann J, Parrington J, Galione A. Two-pore channels form homoand heterodimers. J Biol Chem. 2011;286:37058-62.

17. Pettersen EF, Goddard TD, Huang CC, Couch GS, Greenblatt DM, Meng EC, et al. UCSF Chimera-a visualization system for exploratory research and analysis. J Comput Chem. 2004;25:1605-12.

18. Lin-Moshier Y, Keebler MV, Hooper R, Boulware MJ, Liu X, Churamani D, et al. The two-pore channel (TPC) interactome unmasks isoform-specific roles for TPCs in endolysosomal morphology and cell pigmentation. Proc Natl Acad Sci U S A. 2014;111:13087-92.

19. Yamaguchi S, Jha A, Li Q, Soyombo AA, Dickinson GD, Churamani D, et al. Transient receptor potential mucolipin 1 (TRPML1) and two-pore channels are functionally independent organellar ion channels. J Biol Chem. 2011;286:22934-42.

20. Gomez-Suaga P, Luzon-Toro B, Churamani D, Zhang L, Bloor-Young D, Patel S, et al. Leucine-rich repeat kinase 2 regulates autophagy through a calcium-dependent pathway involving NAADP. Hum Mol Genet. 2012;21:511-25.

21. Lopez J, Dionisio N, Berna-Erro A, Galan C, Salido GM, Rosado JA. Two-pore channel 2 (TPC2) modulates store-operated $\mathrm{Ca}(2+)$ entry. Biochim Biophys Acta. 2012;1823:1976-83.

22. Lam AKM, Galione A, Lai FA, Zissimopoulos S. Hax-1 identified as a two-pore channel (TPC)-binding protein. FEBS Lett. 2013;587:3782-6.

23. Sharp TV, Wang HW, Koumi A, Hollyman D, Endo Y, Ye H, et al. K15 protein of Kaposi's sarcoma-associated herpesvirus is latently expressed and binds to HAX-1, a protein with antiapoptotic function. J Virol. 2002;76:802-16.

24. Zhang J, Guan X, Shah K, Yan J. Lsm 12 is an NAADP receptor and a two-pore channel regulatory protein required for calcium mobilization from acidic organelles. Nat Commun. 2021;12:4739.

25. Webb SE, Kelu JJ, Miller AL. Role of two-pore channels in embryonic development and cellular differentiation. Cold Spring Harb Perspect Biol. 2020;12:a035170.

26. Marchant JS, Patel S. Two-pore channels at the intersection of endolysosomal membrane traffic. Biochem Soc Trans. 2015;43:434-41. 
27. Ramos I, Reich A, Wessel GM. Two-pore channels function in calcium regulation in sea star oocytes and embryos. Development. 2014;141:4598-609.

28. Grimm C, Holdt LM, Chen CC, Hassan S, Muller C, Jors S, et al. High susceptibility to fatty liver disease in two-pore channel 2-deficient mice. Nat Commun. 2014;5:4699.

29. Sun W, Yue J. TPC2 mediates autophagy progression and extracellular vesicle secretion in cancer cells. Exp Cell Res. 2018;370:478-89.

30. Hamilton A, Zhang Q, Salehi A, Willems M, Knudsen JG, Ringgaard AK, et al. Adrenaline stimulates glucagon secretion by Tpc2-dependent $\mathrm{Ca}^{2+}$ mobilization from acidic stores in pancreatic $\alpha$-Cells. Diabetes. 2018;67:1128-39.

31. Capel RA, Bolton EL, Lin WK, Aston D, Wang Y, Liu W, et al. Two-pore channels (TPC2s) and nicotinic acid adenine dinucleotide phosphate (NAADP) at lysosomal-sarcoplasmic reticular junctions contribute to acute and chronic $\beta$-adrenoceptor signaling in the heart. J Biol Chem. 2015;290:30087-98.

32. Davis LC, Morgan AJ, Galione A. NAADP-regulated two-pore channels drive phagocytosis through endolysosomal $\mathrm{Ca}^{2+}$ nanodomains, calcineurin and dynamin. EMBO J. 2020;39:e104058.

33. Favia A, Desideri M, Gambara G, D'Alessio A, Ruas M, Esposito B, et al. VEGF-induced neoangiogenesis is mediated by NAADP and two-pore channel-2-dependent $\mathrm{Ca}^{2+}$ signaling. Proc Natl Acad Sci U S A. 2014;111:E4706-15.

34. Davis LC, Morgan AJ, Chen JL, Snead CM, Bloor-Young D, Shenderov E, et al. NAADP activates two-pore channels on T cell cytolytic granules to stimulate exocytosis and killing. Curr Biol. 2012;22:2331-7.

35. Sakurai Y, Kolokoltsov AA, Chen CC, Tidwell MW, Bauta WE, Klugbauer N, et al. Ebola virus. Two-pore channels control Ebola virus host cell entry and are drug targets for disease treatment. Science. 2015;347:995-8.

36. Millet JK, Whittaker GR. Host cell entry of Middle East respiratory syndrome coronavirus after twostep, furin-mediated activation of the spike protein. Proc Natl Acad Sci U S A. 2014;111:15214-9.

37. Gunaratne GS, Yang Y, Li F, Walseth TF, Marchant JS. NAADP-dependent $\mathrm{Ca}^{2+}$ signaling regulates Middle East respiratory syndrome-coronavirus pseudovirus translocation through the endolysosomal system. Cell Calcium. 2018;75:30-41.

38. Ou X, Liu Y, Lei X, Li P, Mi D, Ren L, et al. Characterization of spike glycoprotein of SARS-CoV-2 on virus entry and its immune cross-reactivity with SARS-CoV. Nat Commun. 2020;11:1620.

39. Khan N, Halcrow PW, Lakpa KL, Afghah Z, Miller NM, Dowdy SF, et al. Two-pore channels regulate Tat endolysosome escape and Tat-mediated HIV-1 LTR transactivation. FASEB J. 2020;34:4147-62.

40. Hockey LN, Kilpatrick BS, Eden ER, Lin-Moshier Y, Brailoiu GC, Brailoiu E, et al. Dysregulation of lysosomal morphology by pathogenic LRRK2 is corrected by TPC2 inhibition. J Cell Sci. 2015;128:232-8.

41. Tong BCK, Wu AJ, Huang AS, Dong R, Malampati S, Iyaswamy A, et al. Lysosomal TPCN (two pore segment channel) inhibition ameliorates beta-amyloid pathology and mitigates memory impairment in Alzheimer disease. Autophagy. 2021; [Epub ahead of print].

42. Garcia-Rua V, Otero MF, Lear PV, Rodriguez-Penas D, Feijoo-Bandin S, Noguera-Moreno T, et al. Increased expression of fatty-acid and calcium metabolism genes in failing human heart. PLoS One. 2012;7:e37505.

43. Riva L, Yuan S, Yin X, Martin-Sancho L, Matsunaga N, Pache L, et al. Discovery of SARS-CoV-2 antiviral drugs through large-scale compound repurposing. Nature. 2020;586:113-9.

44. Netcharoensirisuk P, Abrahamian C, Tang R, Chen CC, Rosato AS, Beyers W, et al. Flavonoids increase melanin production and reduce proliferation, migration and invasion of melanoma cells by blocking endolysosomal/melanosomal TPC2. Sci Rep. 2021;11:8515.

45. Muller M, Gerndt S, Chao YK, Zisis T, Nguyen ONP, Gerwien A, et al. Gene editing and synthetically accessible inhibitors reveal role for TPC2 in HCC cell proliferation and tumor growth. Cell Chem Biol. 2021;28:1119-31.e27. 
46. Jiang Y, Zhou Y, Peng G, Tian H, Pan D, Liu L, et al. Two-pore channels mediated receptor-operated $\mathrm{Ca}^{2+}$ entry in pulmonary artery smooth muscle cells in response to hypoxia. Int J Biochem Cell Biol. 2018;97:28-35.

47. D’Amore A, Hanbashi AA, Di Agostino S, Palombi F, Sacconi A, Voruganti A, et al. Loss of two-pore channel 2 (TPC2) expression increases the metastatic traits of melanoma cells by a mechanism involving the hippo signalling pathway and store-operated calcium entry. Cancers (Basel). 2020;12:2391.

48. Aley PK, Mikolajczyk AM, Munz B, Churchill GC, Galione A, Berger F. Nicotinic acid adenine dinucleotide phosphate regulates skeletal muscle differentiation via action at two-pore channels. Proc Natl Acad Sci U S A. 2010;107:19927-32.

49. Notomi T, Ezura Y, Noda M. Identification of two-pore channel 2 as a novel regulator of osteoclastogenesis. J Biol Chem. 2012;287:35057-64.

50. Zhang ZH, Lu YY, Yue J. Two pore channel 2 differentially modulates neural differentiation of mouse embryonic stem cells. PLoS One. 2013;8:e66077.

51. Nguyen ONP, Grimm C, Schneider LS, Chao YK, Atzberger C, Bartel K, et al. Two-pore channel function is crucial for the migration of invasive cancer cells. Cancer Res. 2017;77:1427-38.

52. Hwangbo C, Park J, Lee JH. mda-9/Syntenin protein positively regulates the activation of Akt protein by facilitating integrin-linked kinase adaptor function during adhesion to type I collagen. J Biol Chem. 2011;286:33601-12.

53. Goel HL, Mercurio AM. VEGF targets the tumour cell. Nat Rev Cancer. 2013;13:871-82.

54. Itatani Y, Kawada K, Yamamoto T, Sakai Y. Resistance to anti-angiogenic therapy in cancer-alterations to anti-VEGF pathway. Int J Mol Sci. 2018;19:1232.

55. Chen Y, Chen JC, Tseng SH. Tetrandrine suppresses tumor growth and angiogenesis of gliomas in rats. Int J Cancer. 2009;124:2260-9.

56. Ma JW, Zhang Y, Li R, Ye JC, Li HY, Zhang YK, et al. Tetrandrine suppresses human glioma growth by inhibiting cell survival, proliferation and tumour angiogenesis through attenuating STAT3 phosphorylation. Eur J Pharmacol. 2015;764:228-39.

57. Gao JL, Ji X, He TC, Zhang Q He K, Zhao Y, et al. Tetrandrine suppresses cancer angiogenesis and metastasis in 4T1 tumor bearing mice. Evid Based Complement Alternat Med. 2013;2013:265061.

58. Pafumi I, Festa M, Papacci F, Lagostena L, Giunta C, Gutla V, et al. Naringenin impairs two-pore channel 2 activity and inhibits VEGF-induced angiogenesis. Sci Rep. 2017;7:5121.

59. Li Y, Schon C, Chen CC, Yang Z, Liegl R, Murenu E, et al. TPC2 promotes choroidal angiogenesis and inflammation in a mouse model of neovascular age-related macular degeneration. Life Sci Alliance. 2021;4:e202101047.

60. Huang T, Song X, Yang Y, Wan X, Alvarez AA, Sastry N, et al. Autophagy and hallmarks of cancer. Crit Rev Oncog. 2018;23:247-67.

61. Singh SS, Vats S, Chia AY, Tan TZQ, Deng S, Ong MS, et al. Dual role of autophagy in hallmarks of cancer. Oncogene. 2018;37:1142-58.

62. Pereira GJS, Hirata H, Fimia GM, do Carmo LG, Bincoletto C, Han SW, et al. Nicotinic acid adenine dinucleotide phosphate (NAADP) regulates autophagy in cultured astrocytes. J Biol Chem. 2011;286:27875-81.

63. Pereira GJS, Antonioli M, Hirata H, Ureshino RP, Nascimento AR, Bincoletto C, et al. Glutamate induces autophagy via the two-pore channels in neural cells. Oncotarget. 2017;8:12730-40.

64. Garcia-Rua V, Feijoo-Bandin S, Rodriguez-Penas D, Mosquera-Leal A, Abu-Assi E, Beiras A, et al. Endolysosomal two-pore channels regulate autophagy in cardiomyocytes. J Physiol. 2016;594:3061-77.

65. Lu Y, Hao B, Graeff R, Yue J. NAADP/TPC2/Ca(2+) Signaling inhibits autophagy. Commun Integr Biol. 2013;6:e27595. 
66. Lin PH, Duann P, Komazaki S, Park KH, Li H, Sun M, et al. Lysosomal two-pore channel subtype 2 (TPC2) regulates skeletal muscle autophagic signaling. J Biol Chem. 2015;290:3377-89.

67. Bai XY, Liu YG, Song W, Li YY, Hou DS, Luo HM, et al. Anticancer activity of tetrandrine by inducing prodeath apoptosis and autophagy in human gastric cancer cells. J Pharm Pharmacol. 2018;70:1048-58.

68. Guo Y, Pei X. Tetrandrine-Induced Autophagy in MDA-MB-231 Triple-negative breast cancer cell through the inhibition of PI3K/AKT/mTOR signaling. Evid Based Complement Alternat Med. 2019;2019:7517431.

69. Kou B, Liu W, Xu X, Yang Y, Yi Q, Guo F, et al. Autophagy induction enhances tetrandrine-induced apoptosis via the AMPK/mTOR pathway in human bladder cancer cells. Oncol Rep. 2017;38:3137-43.

70. Kosiniak-Kamysz A, Marczakiewicz-Lustig A, Marcinska M, Skowron M, Wojas-Pelc A, Pospiech E, et al. Increased risk of developing cutaneous malignant melanoma is associated with variation in pigmentation genes and VDR, and may involve epistatic effects. Melanoma Res. 2014;24:388-96.

71. Pho LN, Leachman SA. Genetics of pigmentation and melanoma predisposition. G Ital Dermatol Venereol. 2010;145:37-45.

72. Alharbi AF, Parrington J. The role of genetic polymorphisms in endolysosomal ion channels TPC2 and P2RX4 in cancer pathogenesis, prognosis, and diagnosis: a genetic association in the UK Biobank. NPJ Genom Med. 2021;6:58.

73. Ambrosio AL, Boyle JA, Aradi AE, Christian KA, Di Pietro SM. TPC2 controls pigmentation by regulating melanosome pH and size. Proc Natl Acad Sci U S A. 2016;113:5622-7.

74. Howarth KD, Mirza T, Cooke SL, Chin SF, Pole JC, Turro E, et al. NRG1 fusions in breast cancer. Breast Cancer Res. 2021;23:3.

75. Hodgson A, Swanson D, Tang S, Dickson BC, Turashvili G. Gene fusions characterize a subset of uterine cellular leiomyomas. Genes Chromosomes Cancer. 2020;[Epub ahead of print].

76. Wilkerson PM, Reis-Filho JS. The 11q13-q14 amplicon: clinicopathological correlations and potential drivers. Genes Chromosomes Cancer. 2013;52:333-55.

77. Sugahara K, Michikawa Y, Ishikawa K, Shoji Y, Iwakawa M, Shibahara T, et al. Combination effects of distinct cores in 11q13 amplification region on cervical lymph node metastasis of oral squamous cell carcinoma. Int J Oncol. 2011;39:761-9.

78. Huang X, Godfrey TE, Gooding WE, McCarty KS Jr, Gollin SM. Comprehensive genome and transcriptome analysis of the 11q13 amplicon in human oral cancer and synteny to the 7F5 amplicon in murine oral carcinoma. Genes Chromosomes Cancer. 2006;45:1058-69.

79. Jahidin AH, Stewart TA, Thompson EW, Roberts-Thomson SJ, Monteith GR. Differential effects of two-pore channel protein 1 and 2 silencing in MDA-MB-468 breast cancer cells. Biochem Biophys Res Commun. 2016;477:731-6.

80. Li F, Ji JP, Xu Y, Liu RL. Identification a novel set of 6 differential expressed genes in prostate cancer that can potentially predict biochemical recurrence after curative surgery. Clin Transl Oncol. 2019;21:1067-75.

81. Shivakumar M, Lee Y, Bang L, Garg T, Sohn KA, Kim D. Identification of epigenetic interactions between miRNA and DNA methylation associated with gene expression as potential prognostic markers in bladder cancer. BMC Med Genomics. 2017;10:30.

82. Kocarnik JM, Park SL, Han J, Dumitrescu L, Cheng I, Wilkens LR, et al. Pleiotropic and sex-specific effects of cancer GWAS SNPs on melanoma risk in the population architecture using genomics and epidemiology (PAGE) study. PLoS One. 2015;10:e0120491.

83. Kim S, Chen J, Cheng T, Gindulyte A, He J, He S, et al. PubChem in 2021: new data content and improved web interfaces. Nucleic Acids Res. 2021;49:D1388-95.

84. Salehi B, Fokou PVT, Sharifi-Rad M, Zucca P, Pezzani R, Martins N, et al. The therapeutic potential of naringenin: a review of clinical trials. Pharmaceuticals (Basel). 2019;12:11. 
85. Feng XL, Zhan XX, Zuo LSY, Mo XF, Zhang X, Liu KY, et al. Associations between serum concentration of flavonoids and breast cancer risk among Chinese women. Eur J Nutr. 2021;60:1347-62.

86. Khan N, Afaq F, Mukhtar H. Cancer chemoprevention through dietary antioxidants: progress and promise. Antioxid Redox Signal. 2008;10:475-510.

87. Valko M, Leibfritz D, Moncol J, Cronin MTD, Mazur M, Telser J. Free radicals and antioxidants in normal physiological functions and human disease. Int J Biochem Cell Biol. 2007;39:44-84.

88. Le Marchand L, Murphy SP, Hankin JH, Wilkens LR, Kolonel LN. Intake of flavonoids and lung cancer. J Natl Cancer Inst. 2000;92:154-60.

89. Vitelli Storelli F, Molina AJ, Zamora-Ros R, Fernandez-Villa T, Roussou V, Romaguera D, et al. Flavonoids and the risk of gastric cancer: an exploratory case-control study in the MCC-Spain study. Nutrients. 2019;11:967.

90. Pang X, Fu W, Wang J, Kang, Xu L, Zhao Y, et al. Identification of estrogen receptor $\alpha$ antagonists from natural products via in vitro and in silico approaches. Oxid Med Cell Longev. 2018;2018:6040149.

91. So FV, Guthrie N, Chambers AF, Moussa M, Carroll KK. Inhibition of human breast cancer cell proliferation and delay of mammary tumorigenesis by flavonoids and citrus juices. Nutr Cancer. 1996;26:167-81.

92. Bulzomi P, Bolli A, Galluzzo P, Acconcia F, Ascenzi P, Marino M. The naringenin-induced proapoptotic effect in breast cancer cell lines holds out against a high bisphenol a background. IUBMB Life. 2012;64:690-6.

93. Ayob Z, Mohd Bohari SP, Abd Samad A, Jamil S. Cytotoxic activities against breast cancer cells of local justicia gendarussa crude extracts. Evid Based Complement Alternat Med. 2014;2014:732980.

94. Abaza MSI, Orabi KY, Al-Quattan E, Al-Attiyah RJ. Growth inhibitory and chemo-sensitization effects of naringenin, a natural flavanone purified from Thymus vulgaris, on human breast and colorectal cancer. Cancer Cell Int. 2015;15:46.

95. Chandrika BB, Steephan M, Kumar TRS, Sabu A, Haridas M. Hesperetin and naringenin sensitize HER2 positive cancer cells to death by serving as HER2 tyrosine kinase inhibitors. Life Sci. 2016;160:47-56.

96. Ke JY, Banh T, Hsiao YH, Cole RM, Straka SR, Yee LD, et al. Citrus flavonoid naringenin reduces mammary tumor cell viability, adipose mass, and adipose inflammation in obese ovariectomized mice. Mol Nutr Food Res. 2017;61:1600934.

97. Zhao Z, Jin G, Ge Y, Guo Z. Naringenin inhibits migration of breast cancer cells via inflammatory and apoptosis cell signaling pathways. Inflammopharmacology. 2019;27:1021-36.

98. El-Kersh DM, Ezzat SM, Salama MM, Mahrous EA, Attia YM, Ahmed MS, et al. Anti-estrogenic and antiaromatase activities of citrus peels major compounds in breast cancer. Sci Rep. 2021;11:7121.

99. Woo Y, Shin SY, Hyun J, Lee SD, Lee YH, Lim Y. Flavanones inhibit the clonogenicity of HCT116 cololectal cancer cells. Int J Mol Med. 2012;29:403-8.

100. Song HM, Park GH, Eo HJ, Lee JW, Kim MK, Lee JR, et al. Anti-proliferative effect of naringenin through p38-dependent downregulation of cyclin D1 in human colorectal cancer cells. Biomol Ther (Seoul). 2015;23:339-44.

101. Moreno-Jimenez MR, Lopez-Barraza R, Cervantes-Cardoza V, Perez-Ramirez IF, Reyna-Rojas JA, Gallegos-Infante JA, et al. Mechanisms associated to apoptosis of cancer cells by phenolic extracts from two canned common beans varieties (Phaseolus vulgaris L.). J Food Biochem. 2019;43:e12680.

102. Storniolo CE, Sacanella I, Lamuela-Raventos RM, Moreno JJ. Bioactive compounds of Mediterranean cooked tomato sauce (sofrito) modulate intestinal epithelial cancer cell growth through oxidative stress/arachidonic acid cascade regulation. ACS Omega. 2020;5:17071-7.

103. Arul D, Subramanian P. Naringenin (citrus flavonone) induces growth inhibition, cell cycle arrest and apoptosis in human hepatocellular carcinoma cells. Pathol Oncol Res. 2013;19:763-70. 
104. Lim W, Park S, Bazer FW, Song G. Naringenin-induced apoptotic cell death in prostate cancer cells is mediated via the PI3K/AKT and MAPK signaling pathways. J Cell Biochem. 2017;118:1118-31.

105. Gumushan Aktas H, Akgun T. Naringenin inhibits prostate cancer metastasis by blocking voltage-gated sodium channels. Biomed Pharmacother. 2018;106:770-5.

106. Lou C, Zhang F, Yang M, Zhao J, Zeng W, Fang X, et al. Naringenin decreases invasiveness and metastasis by inhibiting TGF- $\beta$-induced epithelial to mesenchymal transition in pancreatic cancer cells. PLoS One. 2012;7:e50956.

107. Park HJ, Choi YJ, Lee JH, Nam MJ. Naringenin causes ASK1-induced apoptosis via reactive oxygen species in human pancreatic cancer cells. Food Chem Toxicol. 2017;99:1-8.

108. Stompor M, Uram L, Podgorski R. In vitro effect of 8-prenylnaringenin and naringenin on fibroblasts and glioblastoma cells-cellular accumulation and cytotoxicity. Molecules. 2017;22:1092.

109. Chen YY, Chang YM, Wang KY, Chen PN, Hseu YC, Chen KM, et al. Naringenin inhibited migration and invasion of glioblastoma cells through multiple mechanisms. Environ Toxicol. 2019;34:233-9.

110. Lentini A, Forni C, Provenzano B, Beninati S. Enhancement of transglutaminase activity and polyamine depletion in B16-F10 melanoma cells by flavonoids naringenin and hesperitin correlate to reduction of the in vivo metastatic potential. Amino Acids. 2007;32:95-100.

111. Nasr Bouzaiene N, Chaabane F, Sassi A, Chekir-Ghedira L, Ghedira K. Effect of apigenin-7-glucoside, genkwanin and naringenin on tyrosinase activity and melanin synthesis in B16F10 melanoma cells. Life Sci. 2016;144:80-5.

112. Choi J, Lee DH, Jang H, Park SY, Seol JW. Naringenin exerts anticancer effects by inducing tumor cell death and inhibiting angiogenesis in malignant melanoma. Int J Med Sci. 2020;17:3049-57.

113. Chang HL, Chang YM, Lai SC, Chen KM, Wang KC, Chiu TT, et al. Naringenin inhibits migration of lung cancer cells via the inhibition of matrix metalloproteinases-2 and -9. Exp Ther Med. 2017;13:739-44.

114. Shi X, Luo X, Chen T, Guo W, Liang C, Tang S, et al. Naringenin inhibits migration, invasion, induces apoptosis in human lung cancer cells and arrests tumour progression in vitro. J Cell Mol Med. 2021;25:2563-71.

115. Leonardi T, Vanamala J, Taddeo SS, Davidson LA, Murphy ME, Patil BS, et al. Apigenin and naringenin suppress colon carcinogenesis through the aberrant crypt stage in azoxymethane-treated rats. Exp Biol Med (Maywood). 2010;235:710-7.

116. Subramanian P, Arul D. Attenuation of NDEA-induced hepatocarcinogenesis by naringenin in rats. Cell Biochem Funct. 2013;31:511-7.

117. Arul D, Subramanian P. Inhibitory effect of naringenin (citrus flavonone) on $N$-nitrosodiethylamine induced hepatocarcinogenesis in rats. Biochem Biophys Res Commun. 2013;434:203-9.

118. Sabarinathan D, Mahalakshmi P, Vanisree AJ. Naringenin, a flavanone inhibits the proliferation of cerebrally implanted C6 glioma cells in rats. Chem Biol Interact. 2011;189:26-36.

119. Sabarinathan D, Vanisree AJ. Plausible role of naringenin against cerebrally implanted C6 glioma cells in rats. Mol Cell Biochem. 2013;375:171-8.

120. Lian GY, Wang QM, Mak TS, Huang XR, Yu XQ, Lan HY. Inhibition of tumor invasion and metastasis by targeting TGF- $\beta$-Smad-MMP2 pathway with asiatic acid and naringenin. Mol Ther Oncolytics. 2021;20:277-89.

121. Bodduluru LN, Kasala ER, Madhana RM, Barua CC, Hussain MI, Haloi P, et al. Naringenin ameliorates inflammation and cell proliferation in benzo(a)pyrene induced pulmonary carcinogenesis by modulating CYP1A1, NFkB and PCNA expression. Int Immunopharmacol. 2016;30:102-10.

122. Fernandez J, Silvan B, Entrialgo-Cadierno R, Villar CJ, Capasso R, Uranga JA, et al. Antiproliferative and palliative activity of flavonoids in colorectal cancer. Biomed Pharmacother. 2021;143:112241.

123. Erdogan S, Doganlar O, Doganlar ZB, Turkekul K. Naringin sensitizes human prostate cancer cells to paclitaxel therapy. Prostate Int. 2018;6:126-35. 
124. Ling D, Marshall GM, Liu PY, Xu N, Nelson CA, Iismaa SE, et al. Enhancing the anticancer effect of the histone deacetylase inhibitor by activating transglutaminase. Eur J Cancer. 2012;48:3278-87.

125. Zhang FY, Du GJ, Zhang L, Zhang CL, Lu WL, Liang W. Naringenin enhances the anti-tumor effect of doxorubicin through selectively inhibiting the activity of multidrug resistance-associated proteins but not P-glycoprotein. Pharm Res. 2009;26:914-25.

126. Qin L, Jin L, Lu L, Lu X, Zhang C, Zhang F, et al. Naringenin reduces lung metastasis in a breast cancer resection model. Protein Cell. 2011;2:507-16.

127. Zhang F, Dong W, Zeng W, Zhang L, Zhang C, Qiu Y, et al. Naringenin prevents TGF- $\beta 1$ secretion from breast cancer and suppresses pulmonary metastasis by inhibiting PKC activation. Breast Cancer Res. 2016;18:38.

128. Han KY, Chen PN, Hong MC, Hseu YC, Chen KM, Hsu LS, et al. Naringenin attenuated prostate cancer invasion via reversal of epithelial-to-mesenchymal transition and inhibited uPA activity. Anticancer Res. 2018;38:6753-8.

129. Jang BC, Lim KJ, Paik JH, Cho JW, Baek WK, Suh MH, et al. Tetrandrine-induced apoptosis is mediated by activation of caspases and PKC-delta in U937 cells. Biochem Pharmacol. 2004;67:1819-29.

130. Liu T, Men Q Wu G, Yu C, Huang Z, Liu X, et al. Tetrandrine induces autophagy and differentiation by activating ROS and Notch1 signaling in leukemia cells. Oncotarget. 2015;6:7992-8006.

131. Liu T, Zhang Z, Yu C, Zeng C, Xu X, Wu G, et al. Tetrandrine antagonizes acute megakaryoblastic leukaemia growth by forcing autophagy-mediated differentiation. Br J Pharmacol. 2017;174:4308-28.

132. Wu G, Liu T, Li H, Li Y, Li D, Li W. c-MYC and reactive oxygen species play roles in tetrandrine-induced leukemia differentiation. Cell Death Dis. 2018;9:473.

133. Wu Z, Wang G, Xu S, Li Y, Tian Y, Niu H, et al. Effects of tetrandrine on glioma cell malignant phenotype via inhibition of ADAM17. Tumour Biol. 2014;35:2205-10.

134. Zhang Y, Wen YL, Ma JW, Ye JC, Wang X, Huang JX, et al. Tetrandrine inhibits glioma stem-like cells by repressing $\beta$-catenin expression. Int J Oncol. 2017;50:101-10.

135. Ma JW, Zhang Y, Ye JC, Li R, Wen YL, Huang JX, et al. Tetrandrine exerts a radiosensitization effect on human glioma through inhibiting proliferation by attenuating ERK phosphorylation. Biomol Ther (Seoul). 2017;25:186-93.

136. Jiang YW, Cheng HY, Kuo CL, Way TD, Lien JC, Chueh FS, etal. Tetrandrine inhibits human brain glioblastoma multiforme GBM 8401 cancer cell migration and invasion in vitro. Environ Toxicol. 2019;34:364-74.

137. Jin Q Kang C, Soh Y, Sohn NW, Lee J, Cho YH, et al. Tetrandrine cytotoxicity and its dual effect on oxidative stress-induced apoptosis through modulating cellular redox states in Neuro 2a mouse neuroblastoma cells. Life Sci. 2002;71:2053-66.

138. Chen Y, Chen JC, Tseng SH. Effects of tetrandrine plus radiation on neuroblastoma cells. Anticancer Res. 2009;29:3163-71.

139. Tao LJ, Zhou XD, Shen CC, Liang CZ, Liu B, Tao Y, et al. Tetrandrine induces apoptosis and triggers a caspase cascade in U2-OS and MG-63 cells through the intrinsic and extrinsic pathways. Mol Med Rep. 2014;9:345-9.

140. Tian DD, Zhang RX, Wu N, Yuan W, Luo SH, Chen HQ, et al. Tetrandrine inhibits the proliferation of human osteosarcoma cells by upregulating the PTEN pathway. Oncol Rep. 2017;37:2795-802.

141. Sun X, Xu R, Deng Y, Cheng H, Ma J, Ji J, et al. Effects of tetrandrine on apoptosis and radiosensitivity of nasopharyngeal carcinoma cell line CNE. Acta Biochim Biophys Sin (Shanghai). 2007;39:869-78.

142. Lin YJ, Peng SF, Lin ML, Kuo CL, Lu KW, Liao CL, et al. Tetrandrine induces apoptosis of human nasopharyngeal carcinoma NPC-TW 076 cells through reactive oxygen species accompanied by an endoplasmic reticulum stress signaling pathway. Molecules. 2016;21:1353. 
143. Cho HS, Chang SH, Chung YS, Shin JY, Park SJ, Lee ES, et al. Synergistic effect of ERK inhibition on tetrandrine-induced apoptosis in A549 human lung carcinoma cells. J Vet Sci. 2009;10:23-8.

144. Chen Z, Zhao L, Zhao F, Yang G, Wang JJ. Tetrandrine suppresses lung cancer growth and induces apoptosis, potentially via the VEGF/HIF-1alpha/ICAM-1 signaling pathway. Oncol Lett. 2018;15:7433-7.

145. Huang AC, Lien JC, Lin MW, Yang JS, Wu PP, Chang SJ, et al. Tetrandrine induces cell death in SAS human oral cancer cells through caspase activation-dependent apoptosis and LC3-I and LC3-II activationdependent autophagy. Int J Oncol. 2013;43:485-94.

146. Yu FS, Yu CS, Chen JC, Yang JL, Lu HF, Chang SJ, et al. Tetrandrine induces apoptosis via caspase-8, -9, and -3 and poly (ADP ribose) polymerase dependent pathways and autophagy through beclin-1/LC3-I, II signaling pathways in human oral cancer HSC-3 cells. Environ Toxicol. 2016;31:395-406.

147. Lien JC, Lin MW, Chang SJ, Lai KC, Huang AC, Yu FS, et al. Tetrandrine induces programmed cell death in human oral cancer CAL 27 cells through the reactive oxygen species production and caspase-dependent pathways and associated with beclin-1-induced cell autophagy. Environ Toxicol. 2017;32:329-43.

148. Qin R, Shen H, Cao Y, Fang Y, Li H, Chen Q et al. Tetrandrine induces mitochondria-mediated apoptosis in human gastric cancer BGC-823 cells. PLoS One. 2013;8:e76486.

149. Chaudhary P, Vishwanatha JK. c-Jun NH2-terminal kinase-induced proteasomal degradation of c-FLIPL/S and Bcl2 sensitize prostate cancer cells to Fas- and mitochondria-mediated apoptosis by tetrandrine. Biochem Pharmacol. 2014;91:457-73.

150. Liu W, Kou B, Ma ZK, Tang XS, Lv C, Ye M, et al. Tetrandrine suppresses proliferation, induces apoptosis, and inhibits migration and invasion in human prostate cancer cells. Asian J Androl. 2015;17:850-3.

151. Kou B, Liu W, He W, Zhang Y, Zheng J, Yan Y, et al. Tetrandrine suppresses metastatic phenotype of prostate cancer cells by regulating Akt/mTOR/MMP-9 signaling pathway. Oncol Rep. 2016;35:2880-6.

152. Li X, Su B, Liu R, Wu D, He D. Tetrandrine induces apoptosis and triggers caspase cascade in human bladder cancer cells. J Surg Res. 2011;166:e45-51.

153. Zhang Y, Liu W, He W, Zhang Y, Deng X, Ma Y, et al. Tetrandrine reverses epithelial-mesenchymal transition in bladder cancer by downregulating Gli-1. Int J Oncol. 2016;48:2035-42.

154. Xu W, Debeb BG, Lacerda L, Li J, Woodward WA. Tetrandrine, a compound common in Chinese traditional medicine, preferentially kills breast cancer tumor initiating cells (TICs) in vitro. Cancers (Basel). 2011;3:2274-85.

155. Wong VKW, Zeng W, Chen J, Yao XJ, Leung ELH, Wang QQ, et al. Tetrandrine, an activator of autophagy, induces autophagic cell death via PKC- $\alpha$ inhibition and mTOR-dependent mechanisms. Front Pharmacol. 2017;8:351.

156. Wang CH, Yang JM, Guo YB, Shen J, Pei XH. Anticancer activity of tetrandrine by inducing apoptosis in human breast cancer cell line MDA-MB-231 in vivo. Evid Based Complement Alternat Med. 2020;2020:6823520.

157. Chen T, Ji B, Chen Y. Tetrandrine triggers apoptosis and cell cycle arrest in human renal cell carcinoma cells. J Nat Med. 2014;68:46-52.

158. Chen S, Liu W, Wang K, Fan Y, Chen J, Ma J, et al. Tetrandrine inhibits migration and invasion of human renal cell carcinoma by regulating Akt/NF-кB/MMP-9 signaling. PLoS One. 2017;12:e0173725.

159. Yoo SM, Oh SH, Lee SJ, Lee BW, Ko WG, Moon CK, et al. Inhibition of proliferation and induction of apoptosis by tetrandrine in HepG2 cells. J Ethnopharmacol. 2002;81:225-9.

160. Oh SH, Lee BH. Induction of apoptosis in human hepatoblastoma cells by tetrandrine via caspasedependent Bid cleavage and cytochrome c release. Biochem Pharmacol. 2003;66:725-31.

161. Ng LT, Chiang LC, Lin YT, Lin CC. Antiproliferative and apoptotic effects of tetrandrine on different human hepatoma cell lines. Am J Chin Med. 2006;34:125-35. 
162. Liu C, Gong K, Mao X, Li W. Tetrandrine induces apoptosis by activating reactive oxygen species and repressing Akt activity in human hepatocellular carcinoma. Int J Cancer. 2011;129:1519-31.

163. Gong K, Chen C, Zhan Y, Chen Y, Huang Z, Li W. Autophagy-related gene 7 (ATG7) and reactive oxygen species/extracellular signal-regulated kinase regulate tetrandrine-induced autophagy in human hepatocellular carcinoma. J Biol Chem. 2012;287:35576-88.

164. Yu VWL, Ho WS. Tetrandrine inhibits hepatocellular carcinoma cell growth through the caspase pathway and G2/M phase. Oncol Rep. 2013;29:2205-10.

165. Huang T, Xu S, Deo R, Ma A, Li H, Ma K, et al. Targeting the $\mathrm{Ca}^{2+} /$ Calmodulin-dependent protein kinase II by Tetrandrine in human liver cancer cells. Biochem Biophys Res Commun. 2019;508:1227-32.

166. Chang KH, Liao HF, Chang HH, Chen YY, Yu MC, Chou CJ, et al. Inhibitory effect of tetrandrine on pulmonary metastases in CT26 colorectal adenocarcinoma-bearing BALB/c mice. Am J Chin Med. 2004;32:863-72.

167. Chen XL, Ren KH, He HW, Shao RG. Involvement of PI3K/AKT/GSK3beta pathway in tetrandrineinduced G1 arrest and apoptosis. Cancer Biol Ther. 2008;7:1073-8.

168. Wu JM, Chen Y, Chen JC, Lin TY, Tseng SH. Tetrandrine induces apoptosis and growth suppression of colon cancer cells in mice. Cancer Lett. 2010;287:187-95.

169. He BC, Gao JL, Zhang BQ, Luo Q Shi Q, Kim SH, et al. Tetrandrine inhibits Wnt/ $\beta$-catenin signaling and suppresses tumor growth of human colorectal cancer. Mol Pharmacol. 2011;79:211-9.

170. Wu K, Zhou M, Wu QX, Yuan SX, Wang DX, Jin JL, et al. The role of IGFBP-5 in mediating the antiproliferation effect of tetrandrine in human colon cancer cells. Int J Oncol. 2015;46:1205-13.

171. Tsai SC, Wu WC, Yang JS. Tetrandrine inhibits epithelial-mesenchymal transition in IL-6-induced HCT116 human colorectal cancer cells. Onco Targets Ther. 2021;14:4523-36.

172. Zhang H, Xie B, Zhang Z, Sheng X, Zhang S. Tetrandrine suppresses cervical cancer growth by inducing apoptosis in vitro and in vivo. Drug Des Devel Ther. 2018;13:119-27.

173. Zhu R, Liu T, Tan Z, Wu X, Li M, Jiang L, et al. Tetrandrine induces apoptosis in gallbladder carcinoma in vitro. Int J Clin Pharmacol Ther. 2014;52:900-5.

174. Singh K, Dong Q, TimiriShanmugam PS, Koul S, Koul HK. Tetrandrine inhibits deregulated cell cycle in pancreatic cancer cells: differential regulation of p21 1 ip1/Waf1, p $27^{\text {Kip } 1}$ and cyclin D1. Cancer Lett. 2018;425:164-73.

175. Zhang Y, Wang C, Wang H, Wang K, Du Y, Zhang J. Combination of tetrandrine with cisplatin enhances cytotoxicity through growth suppression and apoptosis in ovarian cancer in vitro and in vivo. Cancer Lett. 2011;304:21-32.

176. Favia A, Pafumi I, Desideri M, Padula F, Montesano C, Passeri D, et al. NAADP-dependent Ca(2+) signaling controls melanoma progression, metastatic dissemination and neoangiogenesis. Sci Rep. 2016;6:18925.

177. Faris P, Pellavio G, Ferulli F, Di Nezza F, Shekha M, Lim D, et al. Nicotinic acid adenine dinucleotide phosphate (NAADP) induces intracellular $\mathrm{Ca}^{2+}$ release through the two-pore channel TPC1 in metastatic colorectal cancer cells. Cancers (Basel). 2019;11:542.

178. Correale P, Tagliaferri P, Celio L, Genua G, Montagnani S, Bianco AR. Verapamil upregulates sensitivity of human colon and breast cancer cells to LAK-cytotoxicity in vitro. Eur J Cancer. 1991;27:1393-5.

179. Bruserud O, Pawelec G. Effects of dipyridamole and R-verapamil on in vitro proliferation of blast cells from patients with acute myelogenous leukaemia. Leuk Res. 1993;17:507-13.

180. Bruserud O. Effect of dipyridamole, theophyllamine and verapamil on spontaneous in vitro proliferation of myelogenous leukaemia cells. Acta Oncol. 1992;31:53-8.

181. Yoon H, Kim TW, Shin SY, Park MJ, Yong Y, Kim DW, et al. Design, synthesis and inhibitory activities of naringenin derivatives on human colon cancer cells. Bioorg Med Chem Lett. 2013;23:232-8. 
182. Rebello CJ, Beyl RA, Lertora JJL, Greenway FL, Ravussin E, Ribnicky DM, et al. Safety and pharmacokinetics of naringenin: a randomized, controlled, single-ascending-dose clinical trial. Diabetes Obes Metab. 2020;22:91-8.

183. Tundis R, Loizzo MR, Menichini F, Bonesi M, Colica C, Menichini F. In vitro cytotoxic activity of extracts and isolated constituents of Salvia leriifolia Benth. Against a panel of human cancer cell lines. Chem Biodivers. 2011;8:1152-62.

184. Schutz R, Muller M, Geisslinger F, Vollmar A, Bartel K, Bracher F. Synthesis, biological evaluation and toxicity of novel tetrandrine analogues. Eur J Med Chem. 2020;207:112810.

185. Liu T, Liu X, Li W. Tetrandrine, a Chinese plant-derived alkaloid, is a potential candidate for cancer chemotherapy. Oncotarget. 2016;7:40800-15.

186. Chi M, Evans H, Gilchrist J, Mayhew J, Hoffman A, Pearsall EA, et al. Phosphorylation of calcium/ calmodulin-stimulated protein kinase II at T286 enhances invasion and migration of human breast cancer cells. Sci Rep. 2016;6:33132.

187. Hoffman A, Carpenter H, Kahl R, Watt LF, Dickson PW, Rostas JA, et al. Dephosphorylation of CaMKII at T253 controls the metaphase-anaphase transition. Cell Signal. 2014;26:748-56.

188. Wang J, Guo M, Ma R, Wu M, Zhang Y. Tetrandrine alleviates cerebral ischemia/reperfusion injury by suppressing NLRP3 inflammasome activation via Sirt-1. PeerJ. 2020;8:e9042.

189. Song MY, Wang JX, Sun YL, Han ZF, Zhou YT, Liu Y, et al. Tetrandrine alleviates silicosis by inhibiting canonical and non-canonical NLRP3 inflammasome activation in lung macrophages. Acta Pharmacol Sin. 2021; [Epub ahead of print].

190. Wang J, Yao Z, Lai X, Bao H, Li Y, Li S, et al. Tetrandrine sensitizes nasopharyngeal carcinoma cells to irradiation by inducing autophagy and inhibiting MEK/ERK pathway. Cancer Med. 2020;9:7268-78.

191. Xu XH, Gan YC, Xu GB, Chen T, Zhou H, Tang JF, et al. Tetrandrine citrate eliminates imatinib-resistant chronic myeloid leukemia cells in vitro and in vivo by inhibiting Bcr-Abl/ $\beta$-catenin axis. J Zhejiang Univ Sci B. 2012;13:867-74.

192. Zhu X, Sui M, Fan W. In vitro and in vivo characterizations of tetrandrine on the reversal of P-glycoproteinmediated drug resistance to paclitaxel. Anticancer Res. 2005;25:1953-62.

193. Jin J, Wang FP, Wei H, Liu G. Reversal of multidrug resistance of cancer through inhibition of P-glycoprotein by 5-bromotetrandrine. Cancer Chemother Pharmacol. 2005;55:179-88.

194. Liu ZL, Hirano T, Tanaka S, Onda K, Oka K. Persistent reversal of P-glycoprotein-mediated daunorubicin resistance by tetrandrine in multidrug-resistant human T lymphoblastoid leukemia MOLT-4 cells. J Pharm Pharmacol. 2003;55:1531-7.

195. Wan J, Liu T, Mei L, Li J, Gong K, Yu C, et al. Synergistic antitumour activity of sorafenib in combination with tetrandrine is mediated by reactive oxygen species (ROS)/Akt signaling. Br J Cancer. 2013;109:342-50.

196. Chen Y, Li P, Yang S, Tong N, Zhang J, Zhao X. Tetrandrine enhances the anticancer effects of arsenic trioxide in vitro. Int J Clin Pharmacol Ther. 2014;52:416-24.

197. Mei L, Chen Y, Wang Z, Wang J, Wan J, Yu C, et al. Synergistic anti-tumour effects of tetrandrine and chloroquine combination therapy in human cancer: a potential antagonistic role for p21. Br J Pharmacol. 2015;172:2232-45.

198. Yu M, Liu T, Chen Y, Li Y, Li W. Combination therapy with protein kinase inhibitor H89 and tetrandrine elicits enhanced synergistic antitumor efficacy. J Exp Clin Cancer Res. 2018;37:114.

199. Xu WL, Shen HL, Ao ZF, Chen BA, Xia W, Gao F, et al. Combination of tetrandrine as a potential-reversing agent with daunorubicin, etoposide and cytarabine for the treatment of refractory and relapsed acute myelogenous leukemia. Leuk Res. 2006;30:407-13.

200. Liu W, Zhang J, Ying C, Wang Q, Yan C, Jingyue Y, et al. Tetrandrine combined with gemcitabine and cisplatin for patients with advanced non-small cell lung cancer improve efficacy. Int J Biomed Sci. 2012;8:28-35. 
201. Jin H, Li L, Zhong D, Liu J, Chen X, Zheng J. Pulmonary toxicity and metabolic activation of tetrandrine in CD-1 mice. Chem Res Toxicol. 2011;24:2142-52.

202. Tian Y, Shen S, Jiang Y, Shen Q, Zeng S, Zheng J. CYP3A5 mediates bioactivation and cytotoxicity of tetrandrine. Arch Toxicol. 2016;90:1737-48.

203. Shi JP, Li SX, Ma ZL, Gao AL, Song YJ, Zhang H. Acute and sub-chronic toxicity of tetrandrine in intravenously exposed female BALB/c mice. Chin J Integr Med. 2016;22:925-31.

204. Zhang YX, Liu XM, Wang J, Li J, Liu Y, Zhang H, et al. Inhibition of AKT/FoxO3a signaling induced PUMA expression in response to p53-independent cytotoxic effects of $\mathrm{H} 1$ : a derivative of tetrandrine. Cancer Biol Ther. 2015;16:965-75.

205. Wei N, Sun H, Wang F, Liu G. H1, a novel derivative of tetrandrine reverse P-glycoprotein-mediated multidrug resistance by inhibiting transport function and expression of P-glycoprotein. Cancer Chemother Pharmacol. 2011;67:1017-25.

206. Lin Y, Wang Y, Liu X, Yan J, Su L, Liu X. A novel derivative of tetrandrine (H1) induces endoplasmic reticulum stress-mediated apoptosis and prosurvival autophagy in human non-small cell lung cancer cells. Tumour Biol. 2016;37:10403-13.

207. Naylor E, Arredouani A, Vasudevan SR, Lewis AM, Parkesh R, Mizote A, et al. Identification of a chemical probe for NAADP by virtual screening. Nat Chem Biol. 2009;5:220-6.

208. Jin X, Zhang Y, Alharbi A, Hanbashi A, Alhoshani A, Parrington J. Targeting two-pore channels: current progress and future challenges. Trends Pharmacol Sci. 2020;41:582-94.

209. Simpson WG. The calcium channel blocker verapamil and cancer chemotherapy. Cell Calcium. 1985;6:449-67.

210. Genazzani AA, Mezna M, Dickey DM, Michelangeli F, Walseth TF, Galione A. Pharmacological properties of the $\mathrm{Ca}^{2+}$-release mechanism sensitive to NAADP in the sea urchin egg. Br J Pharmacol. 1997;121:1489-95.

211. Pahor M, Furberg CD. Is the use of some calcium antagonists linked to cancer? Evidence from recent observational studies. Drugs Aging. 1998;13:99-108.

212. Solary E, Bidan JM, Calvo F, Chauffert B, Caillot D, Mugneret F, et al. P-glycoprotein expression and in vitro reversion of doxorubicin resistance by verapamil in clinical specimens from acute leukaemia and myeloma. Leukemia. 1991;5:592-7.

213. Ledwitch KV, Gibbs ME, Barnes RW, Roberts AG. Cooperativity between verapamil and ATP bound to the efflux transporter P-glycoprotein. Biochem Pharmacol. 2016;118:96-108.

214. Muller C, Bailly JD, Goubin F, Laredo J, Jaffrezou JP, Bordier C, et al. Verapamil decreases P-glycoprotein expression in multidrug-resistant human leukemic cell lines. Int J Cancer. 1994;56:749-54.

215. McCarthy M, Auda G, Agrawal S, Taylor A, Backstrom Z, Mondal D, et al. In vivo anticancer synergy mechanism of doxorubicin and verapamil combination treatment is impaired in BALB/c mice with metastatic breast cancer. Exp Mol Pathol. 2014;97:6-15.

216. Shiozaki A, Katsurahara K, Kudou M, Shimizu H, Kosuga T, Ito H, et al. Amlodipine and verapamil, voltage-gated $\mathrm{Ca}^{2+}$ channel inhibitors, suppressed the growth of gastric cancer stem cells. Ann Surg Oncol. 2021;28:5400-11.

217. Maruyama Y, Murohashi I, Nara N, Aoki N. Effects of verapamil on the cellular accumulation of daunorubicin in blast cells and on the chemosensitivity of leukaemic blast progenitors in acute myelogenous leukaemia. Br J Haematol. 1989;72:357-62.

218. Tsuruo T, Iida H, Yamashiro M, Tsukagoshi S, Sakurai Y. Enhancement of vincristine- and adriamycininduced cytotoxicity by verapamil in P388 leukemia and its sublines resistant to vincristine and adriamycin. Biochem Pharmacol. 1982;31:3138-40.

219. Li P, Zhong D, Gong PY. Synergistic effect of paclitaxel and verapamil to overcome multi-drug resistance in breast cancer cells. Biochem Biophys Res Commun. 2019;516:183-8. 
220. Wang X, Wang Z, Wang K, Gao M, Zhang H, Xu X. Metabolomics analysis of multidrug resistance in colorectal cancer cell and multidrug resistance reversal effect of verapamil. Biomed Chromatogr. 2021;35:e4976.

221. Schuldes H, Dolderer JH, Zimmer G, Knobloch J, Bickeboller R, Jonas D, et al. Reversal of multidrug resistance and increase in plasma membrane fluidity in $\mathrm{CHO}$ cells with R-verapamil and bile salts. Eur J Cancer. 2001;37:660-7.

222. Candussio L, Decorti G, Crivellato E, Granzotto M, Rosati A, Giraldi T, et al. Toxicologic and pharmacokinetic study of low doses of verapamil combined with doxorubicin. Life Sci. 2002;71:3109-19.

223. Ries F, Dicato M. Treatment of advanced and refractory breast cancer with doxorubicin, vincristine and continuous infusion of verapamil. A phase I-II clinical trial. Med Oncol Tumor Pharmacother. 1991;8:39-43.

224. Pingsheng F, Tengyue Z, Qiang H, Qiang W, Xin S, Liting Q. Basic and clinical research on the therapeutic effect of intervention in primary liver cancer by targeted intra-arterial verapamil infusion. Cell Biochem Biophys. 2012;62:59-67.

225. Huang J, Zhang T, Ma K, Fan P, Liu Y, Weng C, et al. Clinical evaluation of targeted arterial perfusion of verapamil and chemotherapeutic drugs in interventional therapy of advanced lung cancer. Cancer Chemother Pharmacol. 2013;72:889-96.

226. Fan GF, Pan JJ, Fan PS, Zhang TY, Liu YB, Huang J, et al. The clinical observation of verapamil in combination with interventional chemotherapy in advanced gastric cancer. Eur Rev Med Pharmacol Sci. 2018;22:5508-18. 